\title{
Dynamical Properties of the Molniya Satellite Constellation: Long-Term Evolution of the Semi- Major Axis
}

Jérôme Daquin ( $\nabla$ daquin.jerome@gmail.com )

University of Namur https://orcid.org/0000-0002-8931-7527

Elisa Maria Alessi

IMATI CNR Milano: Istituto di Matematica Applicata e Tecnologie Informatiche Enrico Magenes Consiglio Nazionale delle Ricerche Sezione di Milano

Joseph O'Leary

EOS

\section{Anne Lemaitre}

University of Namur: Universite de Namur

Alberto Buzzoni

Osservatorio di Astrofisica e Scienza dello Spazio di Bologna

\section{Research Article}

Keywords: Molniya orbit, tesseral resonance, lunisolar resonance, hyperbolic sets, space situational awareness

Posted Date: March 16th, 2021

DOI: https://doi.org/10.21203/rs.3.rs-295816/v1

License: (9) This work is licensed under a Creative Commons Attribution 4.0 International License. Read Full License

Version of Record: A version of this preprint was published at Nonlinear Dynamics on July 26th, 2021. See the published version at https://doi.org/10.1007/s11071-021-06708-5. 
Noname manuscript No.

(will be inserted by the editor)

\title{
Dynamical properties of the Molniya satellite constellation: long-term evolution of the semi-major axis
}

\author{
Jérôme Daquin · Elisa Maria Alessi · Joseph \\ O'Leary · Anne Lemaitre · Alberto Buzzoni
}

March 3, 2021

7 Abstract We describe the phase space structures related to the semi-major axis of Molniya-like satellites subject to tesseral and lunisolar resonances. In particular, we dissect the indirect interplay of the critical inclination resonance on the semi-geosynchronous resonance using a hierarchy of more realistic dynamical systems, thus discussing the dynamics beyond the integrable approximation. By introducing ad hoc tractable models averaged over the fast angles, we numerically demarcate the hyperbolic structures organising the long-term dynamics via the computation of finite-time variational indicators. Based on the publicly available two-line elements space orbital data, we identify two satellites, namely M1-69 and M1-87, displaying fingerprints consistent with the dynamics associated to the hyperbolic set. The computations of the associated dynamical maps highlight that the spacecraft are trapped within the hyperbolic tangle.

17 Keywords Molniya orbit - tesseral resonance - lunisolar resonance - hyperbolic sets · space situational awareness

\section{Introduction}

This paper is part of a recent series of papers dedicated to properties and dynamics of Molniya spacecraft $[1,6,29]$ with emphasis on the long-term evolution of the semi-major axis. We approach the problem by studying the long-term and drag-free motion of a test-particle subject to the nonspherical geometry of the Earth and third-body perturbations due to the Sun and the Moon. The metrical Keplerian prototypical values, semi-major axis a (expressed in units of Earth radius, $r_{\mathrm{E}}$ ), eccentricity $e$ and inclination $i$, considered in this work are

$$
œ_{\mathcal{M}}=\left(a_{\mathcal{M}}, e_{\mathcal{M}}, i_{\mathcal{M}}\right) \sim\left(4.16 r_{\mathrm{E}}, 0.7,63.4^{\circ}\right)
$$

Jérôme Daquin

Department of Mathematics (naXys), 61 Rue de Bruxelles, 5000, Namur, Belgium

E-mail: jerome.daquin@unamur.be

Elisa Maria Alessi

Istituto di Matematica Applicata e Tecnologie Informatiche "Enrico Magenes", Consiglio Nazionale delle Ricerche, Via Alfonso Corti 12, 20133 Milano, Italy

Istituto di Fisica Applicata "Nello Carrara", Consiglio Nazionale delle Ricerche, Via Madonna del Piano 10, 50019 Sesto Fiorentino, Italy

Joseph O'Leary

EOS Space Systems Pty. Ltd., Lot Fourteen, McEwin Building. North Terrace, Adelaide, SA 5000, Australia School of Information Technology and Mathematical Sciences, University of South Australia, GPO Box 2471, Adelaide, SA, 5001, Australia

Anne Lemaitre

Department of Mathematics (naXys), 61 Rue de Bruxelles, 5000, Namur, Belgium

Alberto Buzzoni

INAF-OAS, Osservatorio di Astrofisica e Scienza del Spazio, Via P. Gobetti 93/3, 40129 Bologna, Italy 
The zonal geopotential terms are restricted to the second degree $J_{2}$ term. Molniya satellites have a mean motion close to 2 revolutions per day and thus are subject to a 2 : 1 resonant commensurability with the Earth's rotation rate (semi-synchronous orbits). Therefore, 12-hour resonant terms of the geopotential need to be taken into account to model the dynamics. The resonant terms are algebraically computed up to the 4 th degree and order. Being interested in long-term dynamics, it is understood that we deal with the various Hamiltonian contributions averaged over the fast variables, leading to the so-called secular dynamics. The fast timescales are connected to the mean anomaly of the test-particle and the Moon and the Sun, denoted respectively $M, M_{\mathrm{M}}, M_{\mathrm{S}}$. The averaged contributions are introduced as the direct computation of the integral with respect to the fast variables. For the zonal contribution, this averaging is performed in closed form with respect of the eccentricity. The quadrupolar lunisolar perturbations, depending respectively on $M$ and $M_{\mathrm{M}}$ or $M$ and $M_{\mathrm{S}}$ are doubly averaged, also in closed form with respect of the eccentricity. For the resonant contribution of the geopotential, the averaging requires some extra care. First, the averaging is not performed in closed form over the eccentricity. Instead, we employ a truncated series expansion, which, considering the highly eccentric nature of the orbit, is given to 4th order in the eccentricity. Second, the averaging is not performed over the variable $M$ directly, as it would not take into account accurately the resonant dynamics. Instead, this step calls for the introduction of new slow/fast variables taking into account the very resonant nature of the problem [2]. Once those variables are recognised and introduced explicitly, the averaged contribution is obtained in the usual way, i.e., by averaging over the (new) fast variables.

Molniya orbits gather two distinct resonant phenomena ${ }^{1}$, with quite distinct timescales, giving rise to interesting qualitative dynamical behaviour. Firstly, as we mentioned, they are affected by a $2: 1$ geopotential resonance. Secondly, their inclination close to the critical inclination value of $63.4^{\circ}$ place them near a so-called "inclination dependent only" lunisolar resonance [21]. Whilst the first affects the semi-major axis of the orbit, the lunisolar effect manifests primarily on the eccentricity of the orbit, which exhibits large oscillations. These pulsations contribute to modulate the (no-longer constant) coefficients of the tesseral problem; henceforth a coupling and indirect interplay between the two resonances might happen. The seminal contributions regarding the tesseral and lunisolar problems are gathered in $[12,13]$, and in the PhD work of T. Ely [14], later extended to full papers $[15,16]$. F. Delhaise, J. Henrad and A. Morbidelli [12, 13] have focused their study on the eccentricity, inclination and argument of perigee, without paying attention to the behaviour of the semi-major axis. T. Ely connected the resonant problem with large-scale chaos affecting the semi-major axis, including the disturbing effects of the lunisolar perturbation, but for orbital parameters which differ from Molniya orbit [14]. The secular dynamics of Molniya semi-major axis remains partially unexplored.

The first contribution of this paper is to discuss the dynamics of the semi-major axis beyond the integrable picture. For this task, we rely on classical tools from nonlinear dynamics to portray the dynamical structures organising the long-term dynamics (Poincaré section, sections of finitetime variational indicators). The chaotic nature of eccentric and inclined orbits subject to tesseral resonances, often explained through an overlap of nearby resonances [9], has been known for some time in the context of tesseral resonances $[8,10,14]$. Nevertheless, as we will highlight, the extent of chaos affecting the semi-major axis phase space for Molniya satellites is much more limited in the range of $i \sim 63^{\circ}$ compared to the previously studied range of inclinations. In fact, large connected chaotic seas are absent from the dynamics. Yet, hyperbolic orbits still exist and surround the unperturbed separatrix as we will show.

The second contribution of this paper is to reveal the precise effects of this coupling on the dynamics of the semi-major axis. This is achieved via the introduction of several dynamical systems, aiming at isolating gradually the various effects and couplings. The driving principle is to introduce basic dynamical models, with the lowest number of degree-of-freedom (DoF) possible,

1 The force model we employed is discussed with more details in the appendix A. The resonant argument $\omega$ which appears in the expansion of the lunisolar Hamiltonian also appears in higher geopotential zonal terms. In this sense, Molniya orbits gather more than 2 resonant phenomena, being affected by zonal, tesseral and third-body resonances. Nevertheless, the effects of higher zonal terms on the semi-major dynamics are negligible for our study and timescale of interests as we will demonstrate later. 


$$
V(r, \phi, \lambda)=V_{\mathcal{Z}}(r, \phi)+V_{\mathcal{T}}(r, \phi, \lambda),
$$

\footnotetext{
2 Available at space-track.org.

3 NORAD: North American Aerospace Defense Command.
} 
with the zonal and tesseral parts respectively given by

$$
\left\{\begin{array}{l}
V_{\mathcal{Z}}(r, \phi)=\frac{\mu}{r} \sum_{l \geq 2}\left(\frac{r_{\mathrm{E}}}{r}\right)^{l} J_{l, 0} P_{l, 0} \sin \phi \\
V_{\mathcal{T}}(r, \phi, \lambda)=-\frac{\mu}{r} \sum_{l \geq 2} \sum_{m=1}^{l}\left(\frac{r_{\mathrm{E}}}{r}\right)^{l}\left(c_{l, m} \cos m \lambda+s_{l, m} \sin m \lambda\right) P_{l, m}(\sin \phi),
\end{array}\right.
$$

where the vector $(r, \phi, \lambda)$ denote the spherical coordinates (respectively radius, latitude and longitude), $r_{\mathrm{E}}$ denotes the mean Earth's radius, $\mu$ the gravitational parameter of the Earth. The $P_{l, m}$ are the Legendre polynomials of degree $l$ and order $m$. The coefficients $c_{l, m}$ and $s_{l, m}$ are the harmonic coefficients describing Earth's gravity field where we denoted classically $J_{l, 0}=-c_{l, 0}$. Throughout this paper, we denote the Keplerian orbital elements in the usual way as $(a, e, i, \omega, \Omega, M)$ where $a$ denotes the semi-major axis, $e$ the eccentricity, $i$ the inclination, $\omega$ the argument of perigee, $\Omega$ the longitude of the ascending node and $M$ the mean anomaly.

\subsection{The secular zonal part}

The zonal part is dominated by its quadrupole $(l=2)$ term and we therefore truncate $V_{\mathcal{Z}}$ to $l=2$. Being interested by secular properties, the $M$-average of the $J_{2}$ part, defining the secular $J_{2}$ contribution, is computed (in closed form over the eccentricity) using the differential relationships

$$
\mathrm{d} M=\frac{r^{2}}{a \sqrt{1-e^{2}}} \mathrm{~d} f
$$

together with the formula $r=a\left(1-e^{2}\right) /(1+e \cos f)$ (see e.g., [22]):

$$
\bar{V}_{J_{2}}=\frac{1}{2 \pi} \int_{0}^{2 \pi} V_{J_{2}} \mathrm{~d} M=\frac{1}{2 \pi} \int_{0}^{2 \pi} \frac{r^{2}}{a^{2} \sqrt{1-e^{2}}} V_{J_{2}} \mathrm{~d} f .
$$

The classical final expression (5), expressed in terms of the orbital elements, reads

$$
\bar{V}_{J_{2}}=\frac{\mu r_{\mathrm{E}}^{2} J_{2}}{4 a^{3}}\left(1-e^{2}\right)^{-3 / 2}\left(3 \sin ^{2} i-2\right) .
$$

Secular expressions of higher order terms and their effects are given in Appendix A for the sake of completeness. From now on, we drop bars over averaged quantities, bearing in mind that we are dealing with secular functions in this study.

2.2 The secular resonant tesseral part for 12-hour orbits

To compute the resonant secular contribution of the tesseral part

$$
V_{\mathcal{T}}=\sum_{l \geq 2} \mathcal{T}_{l}
$$

with

$$
\mathcal{T}_{l}=\sum_{m=1}^{l}-\frac{\mu}{r}\left(\frac{r_{\mathrm{E}}}{r}\right)^{l}\left(c_{l, m} \cos m \lambda+s_{l, m} \sin m \lambda\right) P_{l, m}(\sin \phi),
$$

we first express it in terms of the orbital elements using a series of formal substitutions. The spherical coordinates are related to the orbital elements by:

$$
\left\{\begin{array}{l}
\cos (\alpha-\Omega)=\cos (\omega+f) / \cos \phi \\
\sin (\alpha-\Omega)=\sin (\omega+f) \cos i / \cos \phi \\
\sin \phi=\sin i \sin (\omega+f)
\end{array}\right.
$$


where $\alpha$ stands for the right ascension of the satellite (again, we refer to [22] for omitted details). The longitude $\lambda$ is written as a function of $\alpha$ and the sidereal time $\theta$ as

$$
\lambda=\alpha-\theta=(\alpha-\Omega)+(\Omega-\theta) .
$$

The sidereal time $\theta$ evolves linearly with time as $\theta=\varpi_{\mathrm{E}} t$, with $\varpi_{\mathrm{E}}=2 \pi /$ sidereal day. Writing the inverse of the radius as

$$
\frac{1}{r}=\frac{1+e \cos f}{a\left(1-e^{2}\right)}
$$

the quantities $\sin f$ and $\cos f$ are then written using their infinite series representation as a function of the mean anomaly $M$ and the Bessel functions $J_{s}$ (see, e.g., [27])

$$
\left\{\begin{array}{l}
\sin f=\lim _{k \rightarrow+\infty} 2 \sqrt{1-e^{2}} \sum_{s=1}^{k} \frac{1}{s} \frac{\mathrm{d}}{\mathrm{d} e} J_{s}(s e) \sin s M, \\
\cos f=\lim _{k \rightarrow+\infty}-e+\frac{2\left(1-e^{2}\right)}{e} \sum_{s=1}^{k} J_{s}(s e) \cos s M .
\end{array}\right.
$$

Applying the aforementioned substitutions into Eq. (7) transforms it into an expression dependent solely on the orbital elements $(a, e, i, \Omega, \omega, M)$ and the sidereal time $\theta$. The angles appear as linear combinations over the rationales of $M, \theta-\Omega$ and $\omega$ [22]. Computing at this stage the brute-force $M$-average to derive the secular tesseral contribution would suppress the dynamical effects of the resonant terms for 12-hour orbits. In fact, in the vicinity of 12-hour orbits, the fast angle

$$
u_{\mathrm{F}}=\theta-\Omega,
$$

combines with the fast variable $M$ as

$$
2 u_{\mathrm{S}}=M-2 u_{\mathrm{F}},
$$

to form a slow varying quantity. Therefore, in the neighborhood of 12-hour orbits, the variable $u_{\mathrm{S}}$ needs to be considered as a slow and independent variable. Dealing therefore with the variables $u_{\mathrm{F}}, u_{\mathrm{S}}, \omega$, there is one fast angle $u_{\mathrm{F}}$ and two slow angles, $\omega$ and $u_{\mathrm{S}}$. The resonant tesseral contribution is therefore obtained by averaging over the fast angle $u_{\mathrm{F}}$ as

$$
\bar{V}_{\mathcal{T}}=\frac{1}{2 \pi} \int_{0}^{2 \pi} V_{\mathcal{T}} \mathrm{d} u_{\mathrm{F}}
$$

The final expression has the form

$$
\bar{V}_{\mathcal{T}}=\sum_{k=\left(k_{1}, k_{2}\right) \in K} h_{k}(a, e, i) \cos \left(\sigma_{k}+k_{1} \lambda_{l m}\right), K \subset \mathbb{Z}^{2},
$$

where

$$
\sigma_{k}=k_{1} u_{\mathrm{S}}+k_{2} \omega,
$$

and $\lambda_{l m}$ is a constant phase-term defined as

$$
\left\{\begin{array}{l}
c_{l, m}=-J_{l m} \cos m \lambda_{l m}, \\
s_{l, m}=-J_{l m} \sin m \lambda_{l m} .
\end{array}\right.
$$

In Tab.1, we provide the final formal expression of the secular resonant terms ${ }^{4}$ for 12 -hour orbits up to $l=4$ appearing in $\bar{V}_{\mathcal{T}}$ for the uplets $k \in K$ with Eq. (12) truncated to $k_{\max }=4$.

4 Note that there are discrepancies with respect to the formulas presented in [8], imputable most probably to a systematic error in the implementation of the eccentricity functions. 


\begin{tabular}{|c|c|c|}
\hline$k=\left(k_{1}, k_{2}\right)$ & $h_{k}(a, e, i)$ & $\sigma_{k}$ \\
\hline $\begin{array}{l}(2,0) \\
(2,2) \\
(2,-2)\end{array}$ & $\begin{array}{c}\frac{-\mu r_{\mathrm{E}}^{2} J_{22} 9 e\left(9 e^{2}-8\right)(\cos i+1)(\cos i-1)}{32 a^{3}} \\
\frac{\mu r_{\mathrm{E}}^{2} J_{22} 3 e\left(e^{2}-8\right)(\cos i+1)^{2}}{64 a^{3}} \\
\frac{\mu r_{\mathrm{E}}^{2} J_{22} e^{3}(\cos i-1)^{2}}{64 a^{3}}\end{array}$ & $\begin{array}{c}M-2 \theta_{S}+2 \Omega \\
M-2 \theta_{S}+2 \Omega+2 \omega \\
M-2 \theta_{S}+2 \Omega-2 \omega\end{array}$ \\
\hline $\begin{array}{l}(2,-3) \\
(2,-1) \\
(2,1) \\
(2,3)\end{array}$ & $\begin{array}{c}\frac{-\mu r_{\mathrm{E}}^{3} J_{32} 5 e^{4}(\cos i-1)^{2} \sin i}{1024 a^{4}} \\
\frac{\mu r_{\mathrm{E}}^{3} J_{32} 15 e^{2}\left(49 e^{2}+22\right)(3 \cos +1)(\cos i-1) \sin i}{\frac{128 a^{4}}{3} J_{32} 15\left(239 e^{4}+128 e^{2}+64\right)(3 \cos i-1)(\cos i+1) \sin i} \\
\frac{\mu r_{\mathrm{E}}^{3} J_{32} 5 e^{2}\left(e^{2}+6\right)(\cos i+1)^{2} \sin i}{128 a^{4}}\end{array}$ & $\begin{array}{l}M-2 \theta_{S}+2 \Omega-3 \omega \\
M-2 \theta_{S}+2 \Omega-\omega \\
M-2 \theta_{S}+2 \Omega+\omega \\
M-2 \theta_{S}+2 \Omega+3 \omega\end{array}$ \\
\hline $\begin{array}{l}(2,0) \\
(2,-2) \\
(2,2)\end{array}$ & $\begin{array}{c}\left.\frac{-\mu r_{\mathrm{E}}^{4} J_{42} 75 e\left(27 e^{2}+8\right)\left(21 \cos ^{2} i \sin ^{2} i-7 \sin ^{2} i-4 \cos ^{2} i+4\right)}{256 a^{5}}{ }^{128 a^{5}} \sin ^{2} i-\cos i+1\right) \\
\frac{\mu r_{\mathrm{E}}^{4} J_{42} 245 e^{3}(\cos i-1)\left(7 \cos \sin _{\mathrm{E}}^{2} J_{42} 15 e\left(33 e^{2} 8\right)(\cos i+1)\left(7 \cos i \sin ^{2} i-\cos i+1\right)\right.}{128 a^{5}} \\
\frac{\mu r_{\mathrm{E}}^{4} J_{42} 35 e^{3}(\cos i+1)^{2} \sin ^{2} i}{512 a^{5}} \\
\frac{-\mu r_{\mathrm{E}}^{4} J_{44} 105\left(65 e^{4}+16 e^{2}+16\right)(\cos i+1)^{3}(\cos i-1)}{32 a^{5}} \\
\frac{-\mu r_{\mathrm{E}}^{4} J_{44} 35 e^{2}\left(2 a^{5}-3\right)(\cos i+1)^{4}}{32 a^{5}}\end{array}$ & $\begin{array}{c}M-2 \theta_{S}+2 \Omega \\
M-2 \theta_{S}+2 \Omega-2 \omega \\
M-2 \theta_{S}+2 \Omega+2 \omega \\
M-2 \theta_{S}+2 \Omega+4 \omega \\
2\left(M-2 \theta_{S}+2 \Omega\right) \\
2\left(M-2 \theta_{S}+2 \Omega\right)+2 \omega \\
2\left(M-2 \theta_{S}+2 \Omega\right)+4 \omega\end{array}$ \\
\hline
\end{tabular}

Table 1 Formal coefficients and resonant angles of the $2: 1$ resonance up to $l_{\max }=m_{\max }=4$ and $\mathcal{O}\left(e^{4}\right)$.

Now that we have at hand the secular disturbing functions, the dynamics are cast into a Hamiltonian framework accounting for the Keplerian central part,

$$
\mathcal{H}=\mathcal{H}_{\text {kep. }}+V_{J_{2}}+\mathcal{T}_{2}
$$

We start by introducing the following canonical resonant coordinates

$$
\begin{cases}I_{1}=-L, & u_{1}=2 \theta-\ell-2 h, \\ I_{2}=G, & u_{2}=g, \\ I_{3}=H-2 L, & u_{3}=h, \\ I_{4}=-2 L-\Gamma, & u_{4}=-\theta,\end{cases}
$$

where $(L, G, H, \ell, g, h)$ denote the classical canonical Delaunay variables related to the Keplerian elements by

$$
\begin{cases}L=\sqrt{\mu a}, & \ell=M, \\ G=L \sqrt{1-e^{2}}, & g=\omega, \\ H=G \cos i, & h=\Omega .\end{cases}
$$

Given that the Hamiltonian (19) is time-dependent, $\dot{\theta}=\varpi_{\mathrm{E}}=2 \pi /$ sidereal day, we supplement the dynamics with $1-$ DoF given by the canonical conjugate variables denoted $(\Gamma, \tau=\theta)$, with 
$\dot{\theta}=\dot{\tau}=\varpi_{\mathrm{E}}$. Those variables enter into the definition of $\left(I_{4}, u_{4}\right)$. The autonomous dynamics (we still note $\mathcal{H}$ the new Hamiltonian) reads

$$
\mathcal{H}=\mathcal{H}+\varpi_{\mathrm{E}} \Gamma .
$$

The Hamiltonian (22) written in terms of the resonant coordinates (20) reduces to a 2-DoF system as both $u_{3}$ and $u_{4}$ are ignorable. Consequently, their conjugate canonical actions, $I_{3}$ and $I_{4}$, are constant over time (i.e., parameters). Note that when $\dot{u}_{2}=0$, the problem is a $1-D o F$ problem and is therefore integrable.

\subsubsection{The integrable approximation}

When $i_{2} \neq 0$, we derive the resonant integrable approximation assuming that the resonances are isolated [26]. It amounts to take into account in (19), besides the action-only dependent part, the harmonic with the largest amplitude (the dominant term, confer [8]). For Molniya's orbital parameters, $\left|h_{2,0}\right|$ is three times larger than $\left|h_{2,2}\right|$, and about $10^{3}$ larger than $\left|h_{2,-2}\right|$. The 1DoF approximation therefore reads

$$
\tilde{\mathcal{H}}=\mathcal{H}_{0}+h_{2,0} \cos \left(u_{1}+2 \lambda_{22}\right) .
$$

The resonance $\dot{u}_{1}=0$, that we denote $\mathcal{R}_{u_{1}}$, occurs for

$$
\varpi_{0}\left(I_{1}\right)=\partial_{I_{1}} \mathcal{H}_{0}=0 .
$$

Solving this equation for fixed $I_{2}, I_{3}$ determined by $œ_{\mathcal{M}}$ expressed to the equivalent semi-major axis, we find

$$
I_{1}=I_{1}^{\star} \leftrightarrow a_{\star}=26,555 \mathrm{~km} .
$$

For a 1-DoF system, orbits coincide with the set of level curves. The phase space is analogue to the classical pendulum dynamics. In fact, a Taylor expansion at order 2 of the Hamiltonian near $I_{1}=I_{1}^{\star}$ reduces the Hamiltonian to

$$
\tilde{\mathcal{H}}=\frac{1}{2} \alpha_{0} J_{1}^{2}+h_{2,0} \cos \left(u_{1}+2 \lambda_{22}\right),
$$

where $J_{1}=I_{1}-I_{1}^{\star}$ and

$$
\alpha_{0}=\left.\partial_{I_{1} I_{1}}^{2} \mathcal{H}_{0}\right|_{I_{1}=I_{1}^{\star}} .
$$

Analytical characteristics of the resonance might be derived from (26). The equilibria are given by the solution of

$$
\left\{\begin{array}{l}
\dot{J}_{1}=-\partial_{u_{1}} \tilde{\mathcal{H}}=h_{2,0} \sin \left(u_{1}+2 \lambda_{22}\right)=0, \\
\dot{u_{1}}=\partial_{J_{1}} \tilde{\mathcal{H}}=\alpha_{0} J_{1}=0
\end{array}\right.
$$

leading to two the equilibrium solutions

$$
\left\{\begin{array}{l}
\mathbf{x}_{s}=\left(0, u_{s}=-2 \lambda_{22}\right) \simeq(0,3.66), \\
\mathbf{x}_{u}=\left(0, u_{s}=\pi-2 \lambda_{22}\right) \simeq(0,0.52) .
\end{array}\right.
$$

The eigensystem of the Jacobian matrix associated to (29) evaluated at the equilibrium solutions shows that $\mathbf{x}_{s}$ is elliptic (stable fixed point) whilst $\mathbf{x}_{u}$ is hyperbolic (unstable fixed point), from which emanates the separatrix. The eigensystem provides the characteristic periods of libration in the harmonic regime as

$$
T_{\text {lib. }}=\frac{2 \pi}{\left|\Im\left(\lambda_{s}\right)\right|} \simeq 1.76 \text { years. }
$$

The pair of eigenvalues $\pm \lambda_{u}$ associated with the unstable point $\mathbf{x}_{u}$ defines an e-folding time $T_{e}$ of

$$
T_{e}=1 /\left|\lambda_{u}\right| \simeq 0.28 \text { year. }
$$




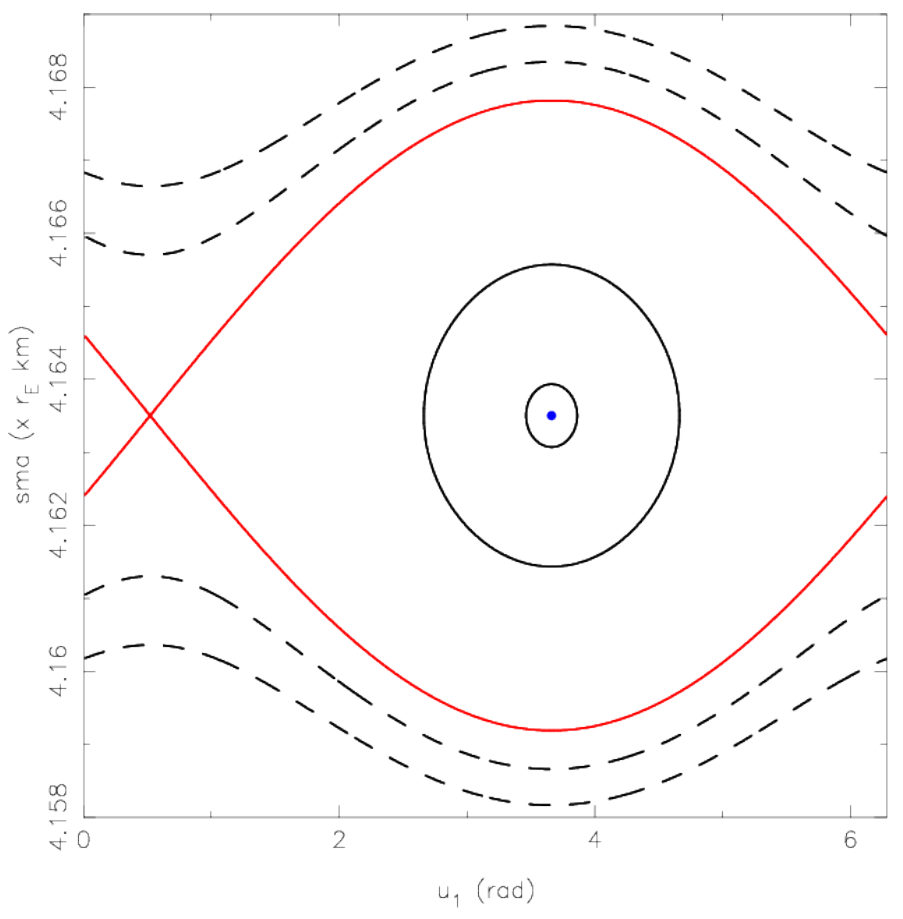

Fig. 1 Phase space of the resonant integrable approximation. The width of the separatrix (red curve) allows excursion of the semi-major axis up to $2 \Delta a=54 \mathrm{~km}$ within the libration domain. The oscillations near the elliptic fixed-point (blue point) have a period of about 1.76 years.

The resonance half-width $\Delta J_{1}$ associated to $(26)$, i.e., the distance between $J_{1}=0$ and the apex of the separatrix (curve associated to the energy level of the unstable equilibria) satisfies

$$
\tilde{\mathcal{H}}\left(\Delta J_{1}, u_{s}\right)=\tilde{\mathcal{H}}\left(0, u_{u}\right),
$$

that is

$$
\frac{1}{2} \alpha_{0} \Delta J_{1}^{2}+h_{2,0}=-h_{2,0} \cdot
$$

Solving the last equality for $\Delta J_{1}$, we find

$$
\Delta J_{1}=2 \sqrt{\frac{\left|h_{2,0}\right|}{\left|\alpha_{0}\right|}} \leftrightarrow \Delta a=27 \mathrm{~km},
$$

When $\dot{u}_{2} \neq 0$, the energy function (19) defines a 2-DoF problem with a multiplet of three resonances with critical angles $u_{1}, u_{1}-u_{2}, u_{1}+u_{2}$. Each isolated resonant problem admits its own pendulum reduction, with the possibility to overlap [9]. Analytical insights might be gained by some simplifications. In fact, let us approximate (19) with the following 2-DoF problem

$$
\mathcal{K}=\frac{1}{2} \alpha_{0} J_{1}^{2}+\varpi_{g} \Gamma+h_{2,0} \cos (\phi)+h_{2,-2} \cos (\phi+2 \tau)+h_{2,2} \cos (\phi-2 \tau),
$$

with $\phi=u_{1}+2 \lambda_{22}$, where we have assumed the rate of variation of $u_{2}=g$ to be ruled by the $\mathcal{H}_{J_{2}}$ part, that is

$$
\dot{u}_{2} \equiv \varpi_{g}=\frac{\partial \mathcal{H}_{J_{2}}}{\partial G}=\left.\frac{3}{4} r_{\mathrm{E}}^{2} J_{2} \frac{\mu^{1 / 2}}{a^{7 / 2}} \frac{5 \cos ^{2} i-1}{\left(1-e^{2}\right)^{2}}\right|_{\aleph_{\mathcal{M}}}
$$


Therefore, $u_{2}$ is a pure time-like variable evolving linearly with time that we rename $\tau$. Using the canonical equations, we find the three resonances centers of $\mathcal{R}_{\phi}, \mathcal{R}_{\phi+2 \tau}, \mathcal{R}_{\phi-2 \tau}$ to be located respectively at

$$
\left\{\begin{array}{l}
c_{\phi}\left(J_{1}\right)=0, \\
c_{\phi+2 \tau}\left(J_{1}\right)=-2 \varpi_{g} / \alpha_{0}, \\
c_{\phi-2 \tau}\left(J_{1}\right)=2 \varpi_{g} / \alpha_{0} .
\end{array}\right.
$$

The mutual distances of the center of the resonances with respect to the center of $\mathcal{R}_{\phi}$,

$$
\left\{\begin{array}{l}
\delta\left(\mathcal{R}_{\phi}, \mathcal{R}_{\phi+2 \tau}\right)=\left|c_{\phi}-c_{\phi+2 \tau}\right|=2\left|\varpi_{g} / \alpha_{0}\right|, \\
\delta\left(\mathcal{R}_{\phi}, \mathcal{R}_{\phi-2 \tau}\right)=\left|c_{\phi}-c_{\phi-2 \tau}\right|=2\left|\varpi_{g} / \alpha_{0}\right|,
\end{array}\right.
$$

are small given the proximity to the critical inclination. The corresponding $\delta a$ amounts to be less than $1 \mathrm{~km}$. Treated as isolated, the resonances $\mathcal{R}_{\phi}, \mathcal{R}_{\phi+2 \tau}, \mathcal{R}_{\phi-2 \tau}$ have the respective half-widths

$$
\left\{\begin{array}{l}
\Delta_{\mathcal{R}_{\phi}}=2 \sqrt{\left|h_{2,0}\right| /\left|\alpha_{0}\right|} \leftrightarrow \Delta_{\mathcal{R}_{\phi}} a=27.5 \mathrm{~km} \\
\Delta_{\mathcal{R}_{\phi+2 \tau}}=2 \sqrt{\left|h_{2,2}\right| /\left|\alpha_{0}\right|} \leftrightarrow \Delta_{\mathcal{R}_{\phi+2 \tau}} a=14.4 \mathrm{~km} \\
\Delta_{\mathcal{R}_{\phi-2 \tau}}=2 \sqrt{\left|h_{2,-2}\right| /\left|\alpha_{0}\right|} \leftrightarrow \Delta_{\mathcal{R}_{\phi-2 \tau}}=0.78 \mathrm{~km}
\end{array}\right.
$$

As inferred from the numerical computation of $h_{2,-2}$, the resonance $\mathcal{R}_{\phi-2 \tau}$ is negligible for practical purposes. Due to the inequalities

$$
\left\{\begin{array}{c}
\Delta_{\mathcal{R}_{\phi}}+\Delta_{\mathcal{R}_{\phi+2 \tau}} \gg \delta\left(\mathcal{R}_{\phi}, \mathcal{R}_{\phi+2 \tau}\right), \\
\Delta_{\mathcal{R}_{\phi}}+\Delta_{\mathcal{R}_{\phi-2 \tau}} \gg \delta\left(\mathcal{R}_{\phi}, \mathcal{R}_{\phi-2 \tau}\right),
\end{array}\right.
$$

Stroboscopic map. The Hamiltonian (35) is a 1-DoF system periodically perturbed. Its phase space can be described by computing the associated Poincaré map, which is, given the periodic nature of the forcing, a stroboscopic mapping [25,32]. Let us denote this mapping by $\mathcal{P}$ and by $\mathcal{V}(0)$ a neighborhood of $J_{1}=0$. By defining the lift and projector operators respectively as

$$
\begin{aligned}
\mathfrak{l}: \mathcal{V}(0) \times[0,2 \pi] & \rightarrow \mathcal{V}(0) \times B \times[0,2 \pi]^{2}, B \subset \mathbb{R} \\
z=\left(J_{1}, u_{1}\right) & \mapsto \mathfrak{l}(z)=\mathbf{x}=\left(J_{1}, \Gamma, u_{1}, \tau\right)
\end{aligned}
$$

and

$$
\begin{aligned}
\mathfrak{p}: \mathcal{V}(0) \times B \times[0,2 \pi]^{2} & \rightarrow \mathcal{V}(0) \times[0,2 \pi] \\
\mathbf{x}=\left(J_{1}, \Gamma, u_{1}, \tau\right) & \mapsto \mathfrak{p}(\mathbf{x})=\left(J_{1}, u_{1}\right),
\end{aligned}
$$

the stroboscopic map is defined as

$$
\begin{aligned}
\mathcal{P}: \mathcal{V}(0) \times[0,2 \pi] & \rightarrow \mathcal{V}(0) \times[0,2 \pi] \\
z & \mapsto \mathcal{P}(z)=z^{\prime}=\mathfrak{p} \circ \Phi^{T_{g}} \circ \mathfrak{l}(z),
\end{aligned}
$$

where $\Phi^{t}$ is the flow at time $t$ associated to (35) and $T_{g}=2 \pi / \varpi_{g}$. Note that the lift is parameterised by the choice of $\tau(0)=g_{0}$. The "dummy" variable $\Gamma$ does not enter into the equations of motion. For Molniya-like spacecraft, $T_{g}$ defines a period of about 100 years (i.e., the order of $10^{4}$ orbital 
revolutions). The mapping $\mathcal{P}$ is constructed numerically based on the numerical propagation of the system (35). Given a value of $g_{0}$, the coordinates of the fixed points of the mapping $\mathcal{P}$ (i.e., the periodic orbits of (35)) are determined using a Newton method. Due to the periodicity

$$
\mathcal{K}\left(J_{1}, u_{1} ; \tau\right)=\mathcal{K}\left(J_{1}, u_{1} ; \tau+\pi\right)
$$

the domain of $g$ can be restricted to $[0, \pi]$. Let us recall that a fixed point $z_{\star}$ of $\mathcal{P}, \mathcal{P}\left(z_{\star}\right)=z_{\star}$, is hyperbolic when the linearisation has at least one eigenvalue with modulus greater than one. In case of complex eigenvalues, the fixed point is elliptic. For $g_{0}=0$, the two fixed points (semi-major axis given in $\mathrm{km}$ ) read as

$$
\left\{\begin{array}{l}
\mathbf{x}_{s}=\left(a, u_{1}\right)=(26554.841,3.662) \\
\mathbf{x}_{u}=\left(a, u_{1}\right)=(26554.850,0.521)
\end{array}\right.
$$

Changing $g_{0}$ alters slightly those coordinates and the slope of the eigenvectors associated to the unstable periodic orbit, which may widen the aperture by a few kilometers. The stable and unstable manifolds associated to an hyperbolic point $z_{\star}$,

$$
\left\{\begin{array}{l}
\mathcal{W}^{s}\left(z_{\star}\right)=\left\{z,\left\|\Phi^{t}(z)-z_{\star}\right\| \rightarrow 0, t \rightarrow+\infty\right\}, \\
\mathcal{W}^{u}\left(z_{\star}\right)=\left\{z,\left\|\Phi^{-t}(z)-z_{\star}\right\| \rightarrow 0, t \rightarrow+\infty\right\},
\end{array}\right.
$$

can be grown by iterating points belonging to the fundamental domain $I \subset E^{s, u}$, where $E^{s, u}$ are respectively the stable and unstable eigenspaces associated to $z_{\star}$ (and derived from the eigensystem analysis). Recall that $\mathcal{W}^{s, u}$ are locally tangent to $E^{s, u}$. In Fig. 2, we show the Poincaré section containing a chaotic zone surrounding the "unperturbed separatrix". A smaller portion of the phase space shows the first lobes associated to the stable manifold. The presence of a chaotic layer brings an important distinguishable qualitative feature to the dynamics: the semi-major axis might display intermittency phenomena (as for geosynchronous orbits, confer [5, 33]). More precisely, for initial conditions in the chaotic layer, the orbit swaps between the "inner-libration" (large range of variation of the semi-major axis), characterised by

$$
\left\langle u_{1}\right\rangle_{\text {lib. }} \simeq 0,
$$

and the "outer-circulation" regime (smaller range of variation of the semi-major axis) for which

$$
\left\langle u_{1}\right\rangle_{\text {circ. }} \not \neq 0,
$$

The alternation takes place when the orbit returns close enough to the hyperbolic saddle $\mathbf{x}_{u}$ which is discussed further in section 3 .

The analysis of the eigensystem associated to the linearisation of $\mathcal{P}$ at the saddle fixed-point is enlightening in deriving the Lyapunov timescale analytically. Let us recall that a Floquet characteristic exponent $\mu$ is a complex number satisfying

$$
\lambda=e^{\mu T_{g}},
$$

where $\lambda$ is an eigenvalue associated to the linearisation $\mathrm{D} \mathcal{P}$ about the fixed point. For the hyperbolic saddle, the two eigenvalues $\left\{\lambda_{1}, \lambda_{2}=1 / \lambda_{1}\right\}$ are real and so are the corresponding $\left\{\mu_{1}, \mu_{2}\right\}$, called in this case the Lyapunov exponents. From

$$
\mu=T_{g}^{-1} \log \lambda
$$

the timescale of $1 / \mu \sim 17$ years is derived for the largest eigenvalue. This timescale has been compared with a brute-force estimation of the maximal Lyapunov exponents $\chi$ based on the variational dynamics (and their associated Lyapunov time $\tau_{\mathcal{L}}=1 / \chi$ ) in the vicinity of the hyperbolic saddle. For hyperbolic orbits, we found Lyapunov times in the range of $15-18$ years, this in very good agreement with the analytical timescale based on $\mathrm{D} \mathcal{P}$.

Limitations of the model $\mathcal{K}$. The model (35) is based on geopotential perturbations only. To build a more realistic model, the lunisolar perturbations, Moon and Sun, need to be included. In its present form, model $\mathcal{K}$ is limited in two ways: 

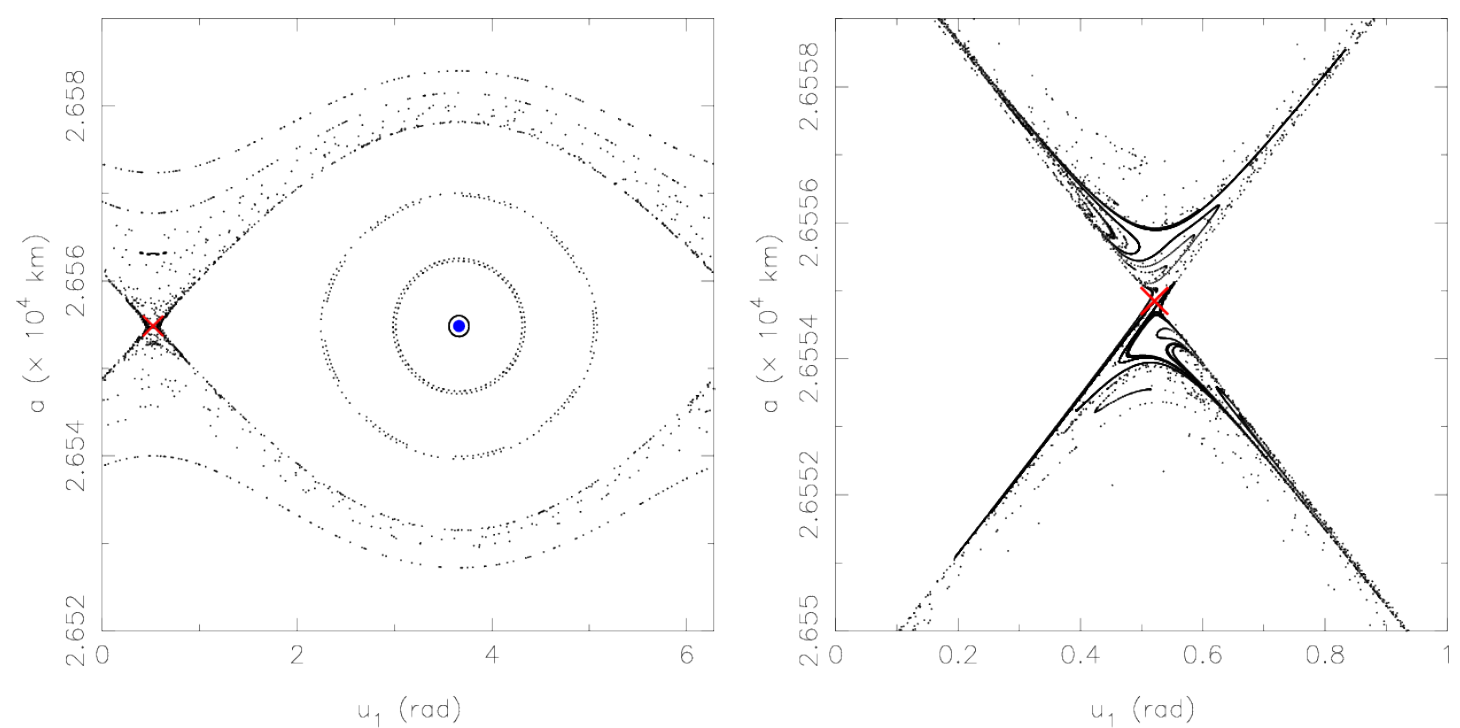

Fig. 2 (Left) Poincaré section associated to (35) computed for $g(0)=0$. The unstable fixed point is labeled with the red cross, the blue circle surrounds the stable periodic orbit. The phase space is similar to the integrable approximation but contains a thin chaotic layer (scattered erratic points) surrounding the unperturbed separatrix. Each considered initial condition has been iterated 100 times under $\mathcal{P}$. (Right) Details of finite pieces of the stable manifold $\mathcal{W}^{s}\left(\mathbf{x}_{u}\right)$.

1. Under the lunisolar effects and due to the proximity to the critical inclination value, the hypothesis that the argument of the perigee $\left(g=u_{2}\right)$ flows linearly with time at a (constant) rate given by the $J_{2}$ effect is violated.

2. The assumption that both the eccentricity and inclination are parameters is no longer true under the influence of the lunisolar perturbation.

Increasing the complexity of the model gradually, we overcome the first limitation by decoupling the equations of motions. We isolate a simplified energy function $\mathcal{L}$ that dictates the time evolution of the argument of perigee, $\dot{g}=\partial_{G} \mathcal{L}$, that we use to form a 6 -dimensional dynamical system with constant eccentricity and inclination. The variables $\left(J_{1}, u_{1}\right)$ are then studied. The second limitation is raised by introducing a 3-DoF Hamiltonian system, where both the eccentricity and inclination vary according to the dynamics.

\section{Secular Hamiltonian including lunisolar effects}

\subsection{The doubly-averaged lunisolar Hamiltonian}

We adopt a simplified sub-model of the quadrupolar doubly-averaged formulation to model the external third-bodies perturbations. The quadrupolar approximation is commonly employed to study medium-Earth orbit dynamics and has already demonstrated its relevance (see e.g., [17, 11]). Starting from the Hamiltonians

$$
\left\{\begin{array}{l}
\mathcal{H}_{\mathrm{M}}=-\frac{\mu_{\mathrm{M}}}{r_{\mathrm{M}}}\left(\frac{r_{\mathrm{M}}}{\left\|\mathbf{r}-\mathbf{r}_{\mathrm{M}}\right\|}-\frac{\mathbf{r} \cdot \mathbf{r}_{\mathrm{M}}}{r_{\mathrm{M}}^{2}}\right), \\
\mathcal{H}_{\mathrm{S}}=-\frac{\mu_{\mathrm{S}}}{r_{\mathrm{S}}}\left(\frac{r_{\mathrm{S}}}{\left\|\mathbf{r}-\mathbf{r}_{\mathrm{S}}\right\|}-\frac{\mathbf{r} \cdot \mathbf{r}_{\mathrm{S}}}{r_{\mathrm{S}}^{2}}\right),
\end{array}\right.
$$

where $\mathbf{r}_{\mathrm{M}}, \mathbf{r}_{\mathrm{S}}$ denote the geocentric vectors of the Moon and the Sun, $r_{\mathrm{M}}, r_{\mathrm{S}}$ the corresponding geocentric distances and $\mu_{\mathrm{M}}, \mu_{\mathrm{S}}$ their respective gravitational parameters, a Legendre-like expansion of $\mathcal{H}_{\mathrm{M}}$ and $\mathcal{H}_{\mathrm{S}}$, truncated to $l=2$ (quadrupolar hypothesis), and averaged over the mean anomalies $\left(M, M_{\mathrm{M}}\right)$ and $\left(M, M_{\mathrm{S}}\right)$ respectively, defines the so-called doubly averaged third-body model. This 
averaging is performed in closed form over the eccentricity. Contrarily to the inner-perturbative part (geopotential), it requires to use the differential relationship

$$
\mathrm{d} M=(1-e \cos E) \mathrm{d} E,
$$

coming from Kepler's equation ( $E$ refers to the eccentric anomaly). The double-averaging

$$
\left\{\begin{array}{l}
\mathcal{H}_{\mathrm{M}}=\frac{1}{(2 \pi)^{2}} \int_{0}^{2 \pi} \int_{0}^{2 \pi} \mathcal{H}_{\mathrm{M}} \mathrm{d} M \mathrm{~d} M_{\mathrm{M}}=\frac{1}{(2 \pi)^{2}} \int_{0}^{2 \pi} \int_{0}^{2 \pi} \mathcal{H}_{\mathrm{M}}(1-e \cos E) \frac{r_{\mathrm{M}}^{2}}{a_{\mathrm{M}}^{2} \sqrt{1-e_{\mathrm{M}}^{2}}} \mathrm{~d} E \mathrm{~d} f_{\mathrm{M}}, \\
\mathcal{H}_{\mathrm{S}}=\frac{1}{(2 \pi)^{2}} \int_{0}^{2 \pi} \int_{0}^{2 \pi} \mathcal{H}_{\mathrm{S}} \mathrm{d} M \mathrm{~d} M_{\mathrm{S}}=\frac{1}{(2 \pi)^{2}} \int_{0}^{2 \pi} \int_{0}^{2 \pi} \mathcal{H}_{\mathrm{S}}(1-e \cos E) \frac{r_{\mathrm{S}}^{2}}{a_{\mathrm{S}}^{2} \sqrt{1-e_{\mathrm{S}}^{2}}} \mathrm{~d} E \mathrm{~d} f_{\mathrm{S}},
\end{array}\right.
$$

reduces $\mathcal{H}_{\mathrm{M}}$ and $\mathcal{H}_{\mathrm{S}}$ to an expansion of the form

$$
\left\{\begin{array}{l}
\mathcal{H}_{\mathrm{M}}=h_{0}^{\mathrm{M}}(a, e, i)+\sum_{j} h_{j}^{\mathrm{M}}(a, e, i) \cos \phi_{j}^{\mathrm{M}}, \\
\mathcal{H}_{\mathrm{S}}=h_{0}^{\mathrm{S}}(a, e, i)+\sum_{j} h_{j}^{\mathrm{S}}(a, e, i) \cos \phi_{j}^{\mathrm{S}},
\end{array}\right.
$$

where $\phi_{i}^{\mathrm{M}}$ and $\phi_{j}^{\mathrm{S}}$ are permitted linear combinations of $\left(\omega, \Omega, \Omega_{\mathrm{M}}\right)$ and $(\omega, \Omega)$ respectively. Given that the angle $\Omega_{\mathrm{M}}$ does not enter $\phi_{j}^{\mathrm{S}}$, the summations are homogenised by introducing $\phi_{q}$, where

$$
\phi_{q}=q_{1} \omega+q_{2} \Omega+q_{3} \Omega_{\mathrm{M}},
$$

with the convention that $q_{3}=0$ for the permissible solar arguments. The quadrupolar doublyaveraged lunisolar Hamiltonian reads therefore

$$
\mathcal{H}_{\mathrm{MS}}=\mathcal{H}_{\mathrm{M}}+\mathcal{H}_{\mathrm{S}}=\left(h_{0}^{\mathrm{M}}+h_{0}^{\mathrm{S}}\right)+\sum_{q \in \mathcal{Q}}\left(h_{q}^{\mathrm{M}}+h_{q}^{\mathrm{S}}\right) \cos \phi_{q},
$$

with (under the quadrupolar assumption)

$$
\mathcal{Q}=\left\{q \in \mathbb{Z}_{\star}^{3} \mid q_{1} \in\{-2,0,2\},\left(q_{2}, q_{3}\right) \in\{-2,-1,0,1,2\}^{2}\right\} .
$$

For the sake of concision, let us denote $h_{j}^{\mathrm{MS}}=h_{j}^{\mathrm{M}}+h_{j}^{\mathrm{S}}$. The Hamiltonian (52) is in general nonautonomous as time enters through the ecliptic precession of the lunar node, well-approximated by the linear law (Moon's elements are referred to the ecliptic plane)

$$
\Omega_{\mathrm{M}} \simeq \Omega_{\mathrm{M}}(0)+\varpi_{\Omega_{\mathrm{M}}} t
$$

where $2 \pi /\left|\varpi_{\Omega_{\mathrm{M}}}\right|$ defines a period of about 18.6 years. The Moon's inclination to the ecliptic plane is set to $i_{\mathrm{M}}=5^{\circ} 15$. However, as we will see hereafter, the simplified model allowed by Molniya's parameters leads to a model independent of the argument of the Moon and, therefore, to an autonomous model.

\subsection{Model for the time evolution of $\omega$}

The so-called "double resonance model" employed in [34] based on the lunisolar harmonics $\cos 2 g$ and $\cos 2 g \pm h$ have shown to provide a realistic model to capture the time evolution of the argument of perigee. They compared orbits generated using this model against TLEs data on several cases and were able to reproduce qualitatively the time evolution of the argument of perigee on several decades. More recently, [29] advocated that the $\cos h$ term produces a significant contribution to the dynamics of $\omega$, where the reader is referred to Appendix A for further details. We adopt the following 2-DoF Hamiltonian system

$$
\mathcal{L}(G, H, g, h)=\mathcal{L}_{0}(G, H)+\mathcal{L}_{1}(G, H, g, h),
$$




\begin{tabular}{cc}
\hline \hline$h_{\sigma}^{\mathrm{M}}(a, e, i)$ & $\sigma$ \\
$-\frac{15 \mu_{\mathrm{M}} a^{2} e^{2} \sin ^{2} i\left(3 \sin ^{2} \varepsilon-2\right)\left(3 \sin ^{2} i_{\mathrm{M}}-2\right)}{64 a_{\mathrm{M}}^{3} \eta_{\mathrm{M}}^{3}}$ & $2 g$ \\
$-\frac{15 \mu_{\mathrm{M}} a^{2} e^{2}(\cos i+1) \cos \varepsilon \sin i \sin \varepsilon\left(3 \sin ^{2} i_{\mathrm{M}}-2\right)}{32 a_{\mathrm{M}}^{3} \eta_{\mathrm{M}}^{3}}$ & $2 g+h$ \\
$-\frac{15 \mu_{\mathrm{M}} a^{2} e^{2}(\cos i-1) \cos \varepsilon \sin i \sin \varepsilon\left(3 \sin ^{2} i_{\mathrm{M}}-2\right)}{32 a_{\mathrm{M}}^{3} \eta_{\mathrm{M}}^{3}}$ & $2 g-h$ \\
$\frac{3 \mu_{\mathrm{M}} a^{2}\left(3 e^{2}+2\right) \cos i \cos \varepsilon \sin i \sin \varepsilon\left(3 \sin ^{2} i_{\mathrm{M}}-2\right)}{16 a_{\mathrm{M}}^{3} \eta_{\mathrm{M}}^{3}}$ & $h$
\end{tabular}

\begin{tabular}{cc}
\hline \hline$h_{\sigma}^{\mathrm{S}}(a, e, i)$ & $\sigma$ \\
\hline \hline$\frac{15 \mu_{\mathrm{S}} a^{2} e^{2} \sin ^{2} i\left(3 \sin ^{2} \varepsilon-2\right)}{32 a_{\mathrm{S}}^{3} \eta_{\mathrm{S}}^{3}}$ & $2 g$ \\
$\frac{15 \mu_{\mathrm{S}} a^{2} e^{2}(\cos i+1) \cos \varepsilon \sin i \sin \varepsilon}{16 a_{\mathrm{S}}^{3} \eta_{\mathrm{S}}^{3}}$ & $2 g+h$ \\
$\frac{15 \mu_{\mathrm{S}} a^{2} e^{2}(\cos i-1) \cos \varepsilon \sin i \sin \varepsilon}{16 a_{\mathrm{S}}^{3} \eta_{\mathrm{S}}^{3}}$ & $2 g-h$ \\
$\frac{-3 \mu_{\mathrm{S}} a^{2}\left(3 e^{2}+2\right) \cos i \cos \varepsilon \sin i \sin \varepsilon}{8 a_{\mathrm{S}}^{3} \eta_{\mathrm{S}}^{3}}$ & $h$
\end{tabular}

Table 2 Formal expression of the lunar and solar coefficients associated to the harmonics $2 g, 2 g \pm h$ and $h$. The obliquity of the ecliptic with respect to the equatorial plane is $\varepsilon=23^{\circ} 44$. The quantity $i_{\mathrm{M}}$ refers to the inclination of the Moon with respect to the ecliptic plane, $i_{\mathrm{M}}=5^{\circ} 15$.

where

$$
\left\{\begin{array}{l}
\mathcal{L}_{0}=\mathcal{H}_{J_{2}}+h_{0}^{\mathrm{MS}}, \\
\mathcal{L}_{1}=h_{2 g}^{\mathrm{MS}} \cos (2 g)+h_{2 g+h}^{\mathrm{MS}} \cos (2 g+h)+h_{2 g-h}^{\mathrm{MS}} \cos (2 g-h),
\end{array}\right.
$$

to model the time evolution of $\omega$. From the canonical equations derived from (55), we derive the dynamics of $g$. The formal coefficients appearing in (55), expressed using the Keplerian elements, are listed in table 2. The terms $h_{0}^{\mathrm{M}}$ and $h_{0}^{\mathrm{S}}$ refer to the action dependent only terms of $\mathcal{H}_{\mathrm{M}}$ and $\mathcal{H}_{\mathrm{S}}$ and read

$$
\left\{\begin{array}{l}
h_{0}^{\mathrm{M}}=\frac{\mu_{\mathrm{M}} a^{2}\left(3 e^{2}+2\right)\left(3 \sin ^{2} i-2\right)\left(3 \sin ^{2} \varepsilon-2\right)\left(3 \sin ^{2} i_{\mathrm{M}}-2\right)}{64 a_{\mathrm{M}}^{3} \eta_{\mathrm{M}}^{3}} \\
h_{0}^{\mathrm{S}}=-\frac{\mu_{\mathrm{S}} a^{2}\left(3 e^{2}+2\right)\left(3 \sin ^{2} i-2\right)\left(3 \sin ^{2} \varepsilon-2\right)}{32 a_{\mathrm{S}}^{3} \eta_{\mathrm{S}}^{3}}
\end{array}\right.
$$

\subsection{Effects of lunisolar perturbation on the tesseral dynamics}

We investigate now how the lunisolar perturbation affects the hyperbolic structures of the tesseral problem for Molniya parameters.

The basic model dictating the time evolution of $g$ being established by (55), we focus now on two dynamical systems improving the caveats of (35):

1. First, we introduce the differential system in $\mathbb{R}^{6}$ defined by the equations of motion (EoM):

$$
\left\{\begin{array}{l}
\dot{J}_{1}=-\partial_{u_{1}} \mathcal{J}\left(J_{1}, u_{1}, g\right), \\
\dot{u}_{1}=\partial_{J_{1}} \mathcal{J}\left(J_{1}, u_{1}, g\right), \\
\dot{G}=-\partial_{g} \mathcal{L}(G, H, g, h), \\
\dot{g}=\partial_{G} \mathcal{L}(G, H, g, h), \\
\dot{H}=-\partial_{h} \mathcal{L}(G, H, g, h), \\
\dot{h}=\partial_{H} \mathcal{L}(G, H, g, h) .
\end{array}\right.
$$

where $\mathcal{J}$ is defined as,

$$
\mathcal{J}\left(J_{1}, u_{1}, g(t)\right)=\frac{1}{2} \alpha_{0} J_{1}^{2}+\mathcal{T}_{2}\left(u_{1}, g(t)\right)
$$

Here $\mathcal{L}$ is the basic lunisolar Hamiltonian function. For short, we refer to the EoM (56) as model $\mathcal{J}$. 
2. Second, we consider the $3-$ DoF Hamiltonian

$$
\mathcal{S}\left(I_{1}, I_{2}, I_{3}, u_{1}, u_{2}, u_{3}\right)=\mathcal{H}_{\text {kep. }}(L)+\varpi_{\mathrm{E}} \Gamma+\mathcal{T}_{2}\left(L, G, H, u_{1}, g\right)+\mathcal{L}(G, H, g, h),
$$

Let us emphasise that both models are $\pi$-periodic in $g$. Molniya spacecraft have, in general, $g \sim$ $270^{\circ} \pm 20^{\circ}$. For both models, in order to reveal the dynamical template and the possible intersection of hyperbolic structures with the $\left(I_{1}, u_{1}\right)$-plane, we compute the Fast Lyapunov Indicators (FLIs) associated to a $500 \times 500$ Cartesian mesh of initial conditions. The FLI is a variational tool that has been used on almost all astronomical scales, for applied or more theoretical oriented studies (see e.g., [18, 24], and references therein). Let us recall that for an $n$-dimensional autonomous ordinary differential system defined on a open domain $D \subset \mathbb{R}^{n}, \dot{x}=f(x)$, the FLI at time $t$ is derived from the linear map $\mathrm{D}_{x} f$ at a point $x$ :

$$
\begin{aligned}
\mathrm{D}_{x} f: \mathbb{R}^{n} & \rightarrow \mathbb{R}^{n}, \\
w & \mapsto \mathrm{D}_{x} f(x) w,
\end{aligned}
$$

and the associated variational equations

$$
\left\{\begin{array}{l}
\dot{x}=f(x) \\
\dot{w}=\mathrm{D}_{x} f(x) w
\end{array}\right.
$$

as

$$
\operatorname{FLI}(t)=\sup _{0 \leq \tau \leq t} \log (\|w(\tau)\|)
$$

The vector $w \in \mathbb{R}^{n}$ denotes the tangent (or deviation) vector. The computation of the FLIs over resolved grid of initial conditions discriminates efficiently the structures of a given dynamical system, including the stable or unstable manifolds, ordered or chaotic seas. One advantage of the FLI over the characteristic Lyapunov exponent

$$
\lambda(x, w)=\lim _{t \rightarrow+\infty} \frac{1}{t} \log (\|w(t)\|)
$$

is to get rid of the time-average computation, thus speeding the stability determination process. The supremum appearing in (61) removes oscillations of the tangent vector. For regular orbits, the deviation vector grows linearly with time and therefore the FLI on regular KAM tori are characterised by values close to $\log \left(t_{f}\right)$. In hyperbolic regions, the norm of the tangent vector grows exponentially fast, and therefore the FLI display a linear trend surpassing quickly the value taken on KAM objects (see [3], chapter 5, for perturbative estimates).

The parametric dependence on $t$ in $(61)$ is raised after a calibration procedure. In our case, integration of several single orbits showed that $\tau_{f}=20$ years are sufficient to obtain a sharp distinction. With our choice of units, the FLI on regular KAM tori is characterised by the value $\log \left(\tau_{f}\right)=4.99$. The FLIs computation are performed for the vector fields $v_{\mathcal{J}}$ and $v_{\mathcal{S}}$ over a Cartesian discretisation of $\Sigma \subset \mathbb{R}^{2}$, where

$$
\Sigma_{i_{0}}=\left\{\left(I_{1}, I_{2}, I_{3}, u_{1}, u_{2}, u_{3}\right):\left(I_{1}, u_{1}\right) \in D, u_{2}=270^{\circ}, u_{3}=0, I_{2}=0.7, I_{3}\left(i_{0}\right)=H_{\star}-2 L_{\star}\right\},
$$

with

$$
D=\mathcal{V}\left(I_{1}^{\star}\right) \times T, \quad T \subset[0,2 \pi] .
$$

The neighborhood of the resonant action $\mathcal{V}\left(I_{1}^{\star}\right)$ represents typically a range of $70 \mathrm{~km}$ in the semimajor axis. Before commenting more on the results, let us mention a last point regarding the FLIs computation. We restricted the computations of the FLIs over $\Sigma$ forward in time, i.e., on a time interval $\left[0, \tau_{f}\right], \tau_{f}>0$, to obtain "positive in time FLIs", FLIs ${ }^{+}$. It is therefore understood that, 
in the context of the existence of hyperbolic invariants, these computations on $\Sigma$ would reveal the trace of the stable manifolds connected to the hyperbolic objects, i.e., $\Sigma \cap \mathcal{W}^{s}$.

Similarly, backwards in time FLIs computed on $\Sigma$ over $\left[-\tau_{f}, 0\right]$, FLIs ${ }^{-}$, would reveal $\Sigma \cap \mathcal{W}^{u}$ (or the hyperbolic chaotic sea in case of a strongly chaotic system). Both manifolds (henceforth, possible homoclinic or heteroclinic connections given the context), could be displayed on $\Sigma$ by plotting the standard average

$$
\mathrm{FLI}=\frac{1}{2}\left(\mathrm{FLI}^{+}+\mathrm{FLI}^{-}\right),
$$

or any others weighted average (see e.g., $[18,24]$ ). We computed a few of those maps backwards in time, to display the averaged FLI. However, because we are not particularly interested of highlighting homoclinic connections and, more importantly, it does not change the dynamical mechanisms we are interested in, we present hereafter only the forward in time FLI maps (i.e., we display $\mathrm{FLI}^{+}$).

Our numerical campaign is parametric through 4 allowed values of $i_{0}$, namely

$$
i_{0} \in\left\{62.5^{\circ}, 63.4^{\circ}, 64.3^{\circ}, 65.2^{\circ}\right\}
$$

"piercing" the critical inclination value. This choice enters $\Sigma_{i_{0}}$ through

$$
I_{3}\left(i_{0}\right)=L_{\star} \sqrt{1-I_{2}^{2}} \cos i_{0}-2 L_{\star}, \quad I_{2}=0.7 .
$$

Although aware that the precise geometry of the hyperbolic structures depend on the initial phasing $(\omega, \Omega)$, our investigations focus on $(\omega, \Omega)=\left(270^{\circ}, 0\right)$. All the resulting maps of this numerical survey for models $\mathcal{J}$ and $\mathcal{S}$ are reported in Appendix $\mathrm{C}$ to ease the readability. We show hereafter in composite panels only the relevant information for the analysis. From this survey, we observe that:

1. Both models display a saddle-like point in $\Sigma$. This suggests the existence of an unstable periodic orbit (although this invariant has not been computed) for both flows, similar to the unstable periodic orbit we computed for model $\mathcal{K}$. Following this idea, the hyperbolic set (high values of FLIs with yellow color) emerging from the saddle-type structure is very likely to represent the intersections of the stable manifold of the hyperbolic invariant with $\Sigma$. The fine mesh of initial conditions allows to recognise lobes distinctively for model $\mathcal{S}$.

2. The model $\mathcal{J}$ is overall weakly perturbed, and the hyperbolic layer is very close to the unperturbed separatrix.

3. On the contrary, the hyperbolic layer of $\mathcal{S}$ is much more developed. This fact is imputable to the indirect modulation of the coefficients $h_{2,0}(a, e, i)$ and $h_{2, \pm 2}(a, e, i)$ under the lunisolar effects. We therefore see the signature of the lunisolar coupling onto the tesseral problem.

4. In general, the width of the hyperbolic layer along a given line of $u_{1}$ increases with the values of $i_{0}$. For $u_{1}$ within the stable librational domain (say $u_{1} \sim 3.6$ ), the hyperbolic width along the line is about $1 \mathrm{~km}$ large in the semi-major axis $\left(i_{0}=62.5^{\circ}\right)$ up to $\sim 6 \mathrm{~km}$ large when $i_{0}=65.2^{\circ}$. For $u_{1}$ near the saddle, for the same inclination values, the hyperbolic layer foliates a width of about $10 \mathrm{~km}$ in the semi-major axis for $i_{0}=62.5^{\circ}$ and up to $30 \mathrm{~km}$ when $i_{0}=65.2^{\circ}$.

5. Lastly, and more will be commented on that in the following, we notice a growing asymmetry of the hyperbolic layer for increasing values of $i_{0}$. At $i_{0}=65.2^{\circ}$, the hyperbolic layer is clearly more developed for the lower range of semi-major axis.

The dynamics associated with model $\mathcal{S}$ is sketched and summarised within the composite panel in Fig. 3 based on the Hamiltonian model

$$
\mathcal{T}=\frac{1}{2} \Lambda^{2}+\Lambda_{1}+\cos (\rho)+\epsilon \cos \left(\rho+\nu \rho_{1}\right),(\epsilon, \nu) \in \mathbb{R}^{2}, \nu \ll 1 .
$$

For $\epsilon=0, \mathcal{T}$ is integrable and has a saddle structure at $(\Lambda, \rho)=(0, \pi)$. The separatrix has a cat-eye topology with half-width $\Delta=2 \sqrt{2}$. When $\epsilon \neq 0, \epsilon \ll 1$, the resonances $\mathcal{R}_{\rho}$ and $\mathcal{R}_{\rho+\nu \rho_{1}}$, $\nu$-apart, produce a separatrix splitting. The resonant angle of an orbit with initial condition in the hyperbolic set alternates among libration, $\langle\rho\rangle \simeq 0$, and circulation, $\langle\rho\rangle \not 0$. The "projection" of one orbit with initial condition close to the saddle (trapped in the hyperbolic tangle) in the 
space $(\Lambda, \rho)$ shows that the orbit remains mainly guided by the unperturbed separatrix. Under our selected initial condition, when the angle circulates, the action is trapped in the tangle, evolving here in the domain $\Lambda^{-}:=\{\Lambda, \Lambda<0\}$. As soon as the $\langle\rho\rangle \not 0$, the action experiences full homoclinic loops and evolve within $\Lambda=\Lambda^{-} \cup \Lambda^{+}$. This process continues and possibly alternates in the vicinity of the unstable saddle, "kicking" the FLIs.

The dynamics associated to the hyperbolic layer of model $\mathcal{S}$ is similar to the one just described, apart that the coefficients of the respective resonances are slowly modulated in time. This is exemplified for two orbits in the composite panel of Fig. 4, together with macro and micro views of the phase space structures. The orbit immersed within the stable region displays gentle oscillations, whilst the orbit trapped into the hyperbolic layer displays the characteristic intermittency. Let us underline that the hyperbolic orbit, on the 20 year timescale, displays U-turns (i.e., the alternation between libration and circulation regimes of the resonant angle $u_{1}$ ) always directed towards lower semi-major axis, with a timescale of about 1.5 year. The full homoclinic loop takes about 3 years. We integrated the same orbit on a time interval 10 times larger and we noticed the unevenly distribution between upper and lower U-turns, the latter being more frequent. This property is clearly inferred from the thorough inspection and detailed geometry of the hyperbolic foldings near the saddle-like structure. The close-up view of the FLI map (see the magnified region materialised by the green box, Fig. 4) reveals more foldings in the lower part of the chart. Increasing the parametric value of $i_{0}$ makes this property even sharper, as shown in the maps provided in Appendix C. The asymmetry of the foldings emerges from the fact that

$$
\delta\left(i_{0}\right)=\frac{\left|h_{2,0}(a, e, i)\right|}{\left|h_{2,2}(a, e, i)\right|}
$$

becomes larger along a solution $\mathbf{x}(t)=(a(t), e(t), i(t)))$ for initial conditions near $\mathbf{x}_{u}$.

The layer's dependence upon $\omega_{0}$, for model $\mathcal{S}$, is shown for the fixed value of $u_{1}=3.6$ in the last map of the composite plot of Fig. 4 (bottom right). It reveals a much wider width (roughly speaking on the order of $10 \mathrm{~km}$ in the semi-major axis) for $\omega \in[\pi, 3 \pi / 2]$, with petals structures. For $\omega \in[3 \pi / 2, \pi]$, the hyperbolic structure is much simpler to apprehend. For Molniya's typical variation of $\omega \sim 270^{\circ} \pm 20^{\circ}$, this corresponds to the rectangle materialized with white dashed lines.

\section{Connections and links with the dynamics of M1-69 and M1-87}

On inspecting the extracted semi-major axis using M1-69 and M1-87 TLE data, we notice that they display intermittency phenomena on their semi-major axis ${ }^{5}$ as repeated in Fig. 5 and Fig. 6. The figures also incorporate the time evolution of the resonant angle $u_{1}$. The relevant part of the data, in the light of the oscillating models previously derived, cover in both cases at least 2 decades. Both data contain a transitory period, possibly remnants of unknown manoeuvres. For M1-87, after the epoch corresponding to mean Julian day (MJD) of $5.7 \times 10^{4}$, the satellite experienced a significant semi-major axis reduction. We will not pay attention to this part of the data. In the exploitable window, the resonant signature, consisting of alternation between libration and circulation, is well-marked and in accordance with the U-turns intermittent semi-major axis variations. In both cases, the intermittency U-turns take place for $a \sim 26,550 \mathrm{~km}$, compatible with the locations of the hyperbolic foldings we located with model $\mathcal{S}$ close to the saddle. It is worth mentioning that M1-69 has been left untouched in [34], as judged to "locate in the vicinity of this separatrix". Below, we give more credit to this claim, and we show that it is also the case for its cousin M1-87. At the light of the dynamical mechanisms presented in section 3 and the fingerprints just described, it is tempting to say that both satellites evolve within the hyperbolic layer. To give more weight to this claim, we performed the following steps:

5 We extracted the mean semi-major axis (in the sense of the underlying SGP4 theory) from the TLEs by following the "un-Kozai" mean-motion procedure (one step iterative method) presented in [19], section 6. See also [30], Eq. (7) or [20], appendix B, section A. 

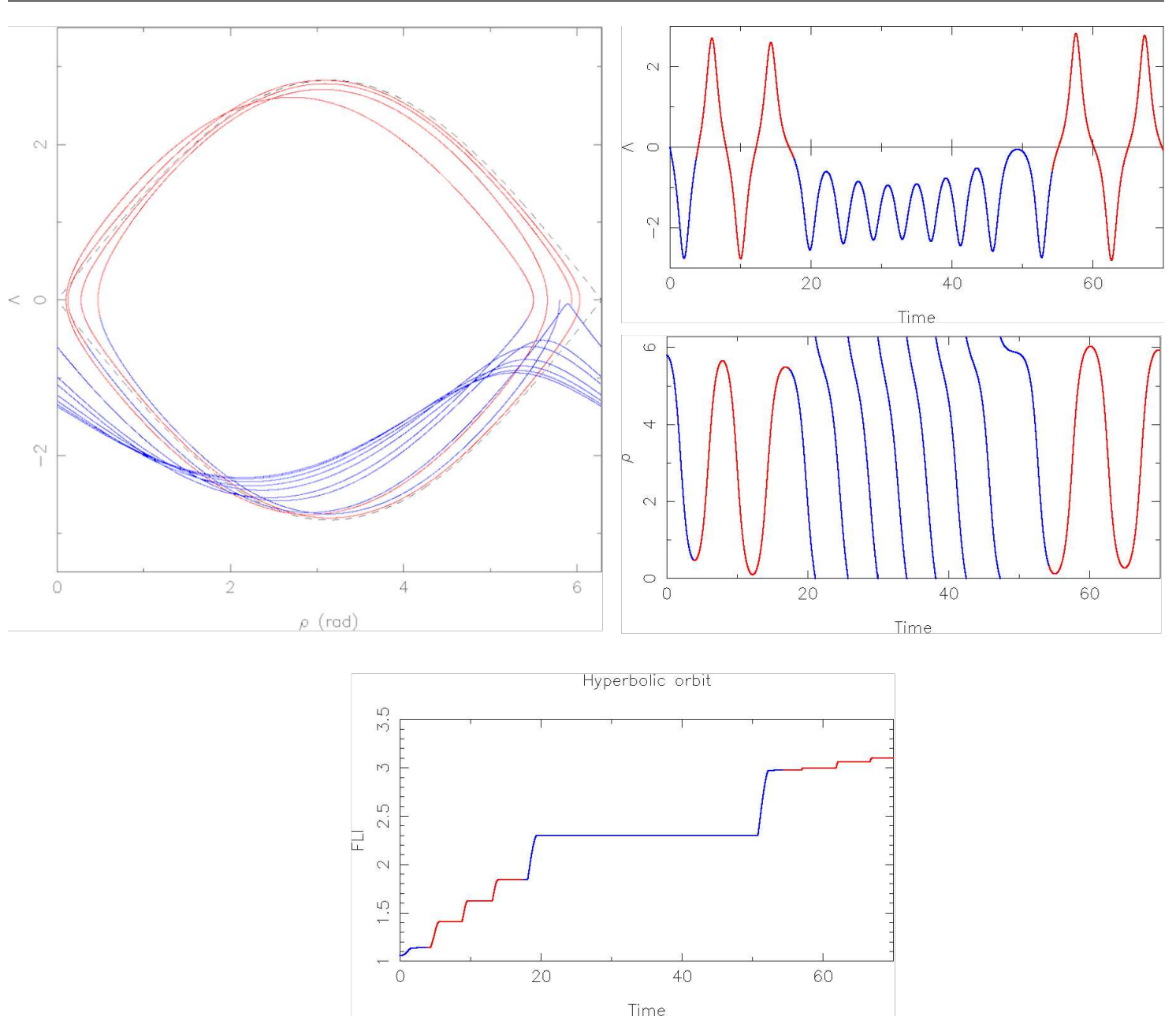

Fig. 3 Composite plot illustrating the mechanisms of the intermittency phenomena. The dashed black line represents the separatrix of the integrable model $\mathcal{T}$. Realisations of the stable and unstable manifolds, for $\epsilon \neq 0, \epsilon \ll 1$, are not shown for the sake of readability. One hyperbolic orbit trapped in the hyperbolic tangle is highlighted in the phase space, with a color code depending on the regime of the resonant angle. When the resonant angle circulates (blue color), $\langle\rho\rangle \not 0$, the action takes negative $\Lambda$ 's. When the angle librates (red color), $\langle\rho\rangle \simeq 0$, the action variable performs the full homoclinic loop and exhibit larger variations. Alternation between circulation and librations with passage close to the saddle contribute to breaks the plateau structure of the FLI and produces jumps in the FLI.

1. At epochs $t_{\star}$ corresponding to the apex of the first U-turns, we extract from the TLEs the corresponding orbital parameters and we record the values of $\left(a^{\star}, u_{1}^{\star}\right)$. For case M1-69, we selected $t_{\star}=50,418.06$ (MJD), leading to the "instantaneous" elements

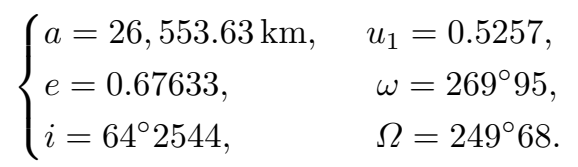

For M1-87, we selected $t_{\star}=53,433.24$ (MJD), for which the sets of computed elements reads

$$
\begin{cases}a=26,550.06 \mathrm{~km}, & u_{1}=0.4749, \\ e=0.6582, & \omega=262^{\circ} 68, \\ i=64^{\circ} 1995, & \Omega=223^{\circ} 01 .\end{cases}
$$

2. We compute the dynamical structures with the FLIs for the vector field $v_{\mathcal{S}}$, using as parameters and phasing for $\Sigma$ those extracted from the respective TLE at epoch $t_{\star}$.

3. On the obtained dynamical maps, the point of coordinates $\left(a\left(t_{\star}\right), u_{1}\left(t_{\star}\right)\right)$ is spotted.

The obtained dynamics maps shown in Fig. 7 convincingly demonstrate that the satellites reside within the hyperbolic tangle. 

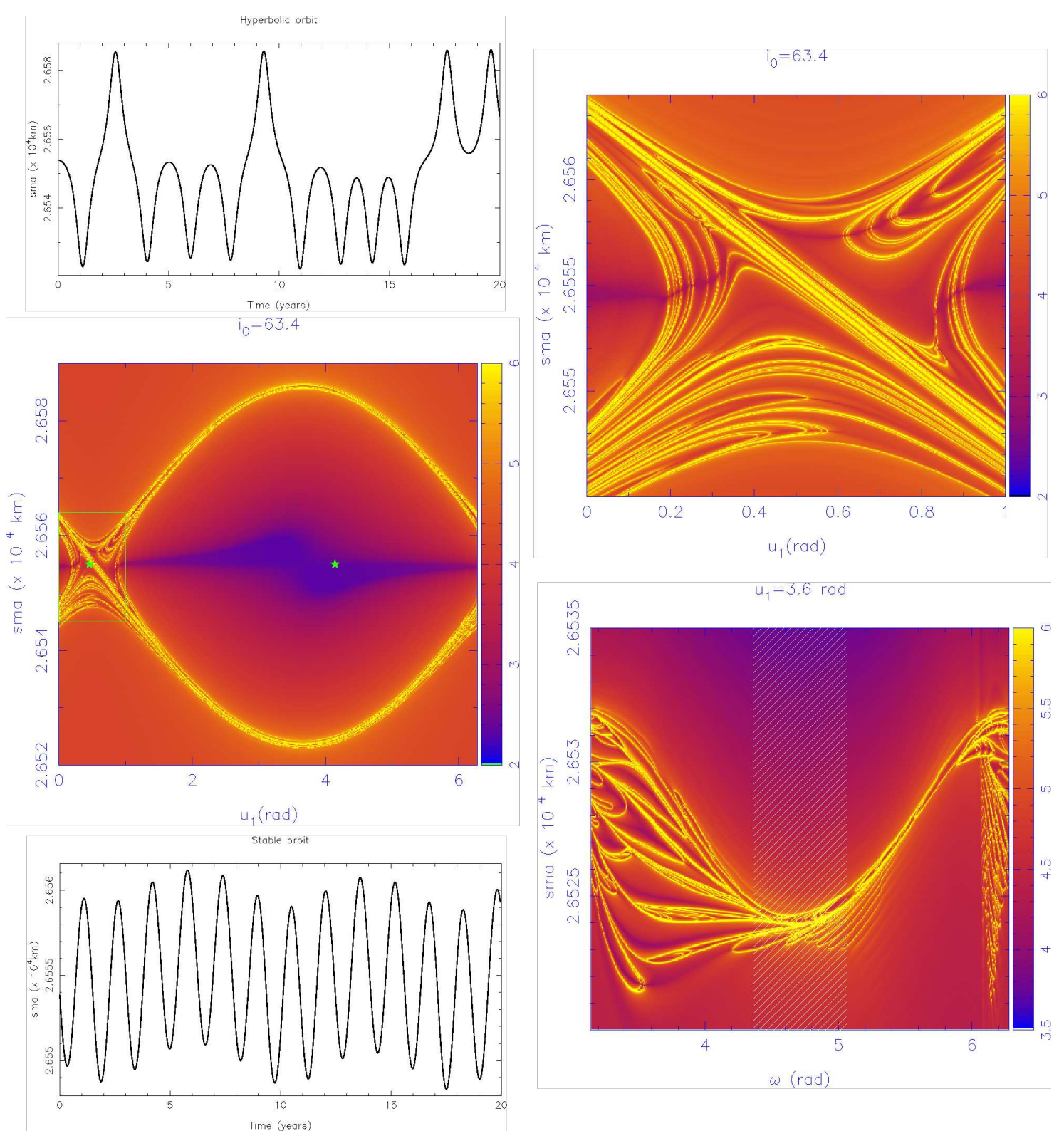

Fig. 4 Composite plot highlighting the main features of Molniya semi-major axis dynamics. The global FLI map and a magnified portion near the saddle-like structure detail the hyperbolic structure. Initial conditions within the hyperbolic layer display intermittency phenomena, whilst stable orbits display regular oscillations. This is exemplified for two orbits whose initial conditions are labeled with the green stars. The width of the layer, for a fixed $u_{1}$ but varying $\omega$, might exhibit a complex geometry. For Molniya's prototypical range of values of $\omega$, materialised by the white shaded-line region around $\omega=270^{\circ}$, the width is limited to a few kilometer in the semi-major axis only. See text for details.

\section{Conclusions}

The constructed dynamical models and their analysis allowed us to deepen the understanding of Molniya's semi-major axis dynamics. The hyperbolic structures organising the phase space have been portrayed via variational indicators through a series of compact, tractable and realistic secular models. The effect of lunisolar perturbations, on the 20 years timescale, needs to be taken into account to reconstruct the correct dynamical template. In fact, the induced modulations of the eccentricity and inclination contribute sufficiently to change the "parameters" of the tesseral problem; the coefficients we denoted by $h_{2,0}$ and $h_{2, \pm 2}$. We connected the 20 year long fingerprints of two satellites, Molniya 1-69 and Molniya 1-87, with the hyperbolic layer surrounding the unper- 
M1-69
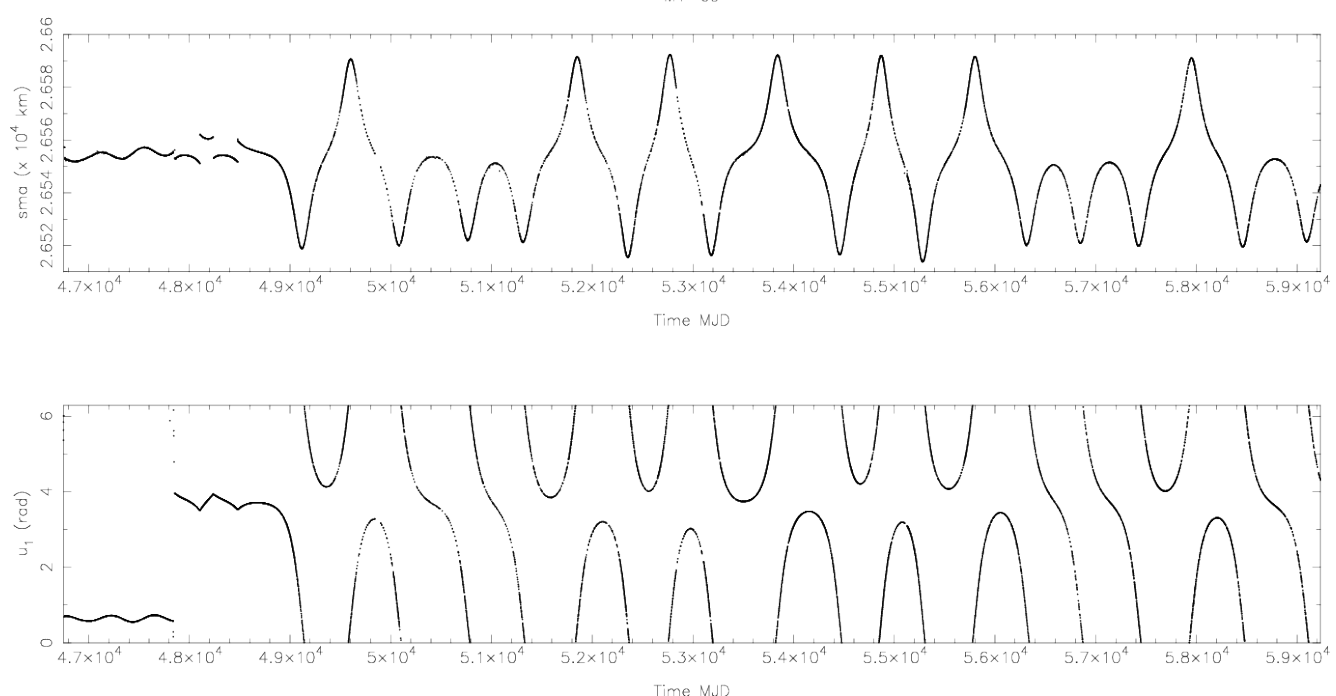

Fig. 5 Time history of the semi-major axis and resonant angle $u_{1}$ extracted from the TLE data for the satellite M1-69.
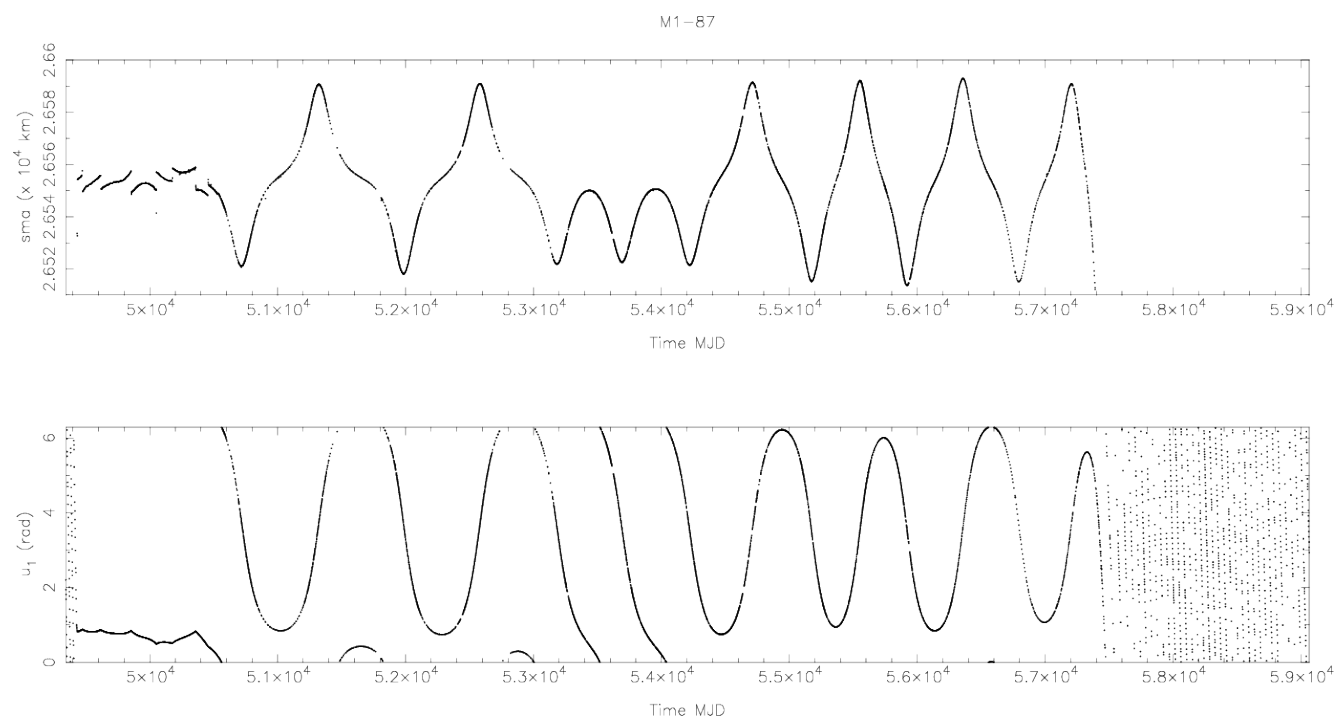

Fig. 6 Time history of the semi-major axis and resonant angle $u_{1}$ extracted from the TLE data for the satellite M1-87

turbed cat-eye separatrix. This hyperbolic layer, in absence of lunisolar perturbations, would be too thin to sustain the dynamical signatures visible at the publicly available data level. By computing their associated dynamical maps, we provided evidence that the two satellites are trapped within the hyperbolic tangle. The secular dynamics umbrella provided a reliable and robust mold to approach and explain the semi-major axis patterns extracted from the TLE space datasets. As far as we are aware, this result is the first report of long time scale hyperbolicity corroborated by pseudo-observations in the near-Earth space environment.

\section{Acknowledgments}

J.D. is a postdoctoral researcher of the "Fonds de la Recherche Scientifique" - FNRS. J.D. acknowledges discussions with Florent Deleflie, Denis Hautesseres, Alexis Petit and David Vallado about the TLEs data, and discussions that have followed from the CNES COMET-ORB workshop on "Uncertainty Quantification in Orbit Propagation", Feb. 9-10, 2021. J.D. acknowledges 
Molniya M1-69

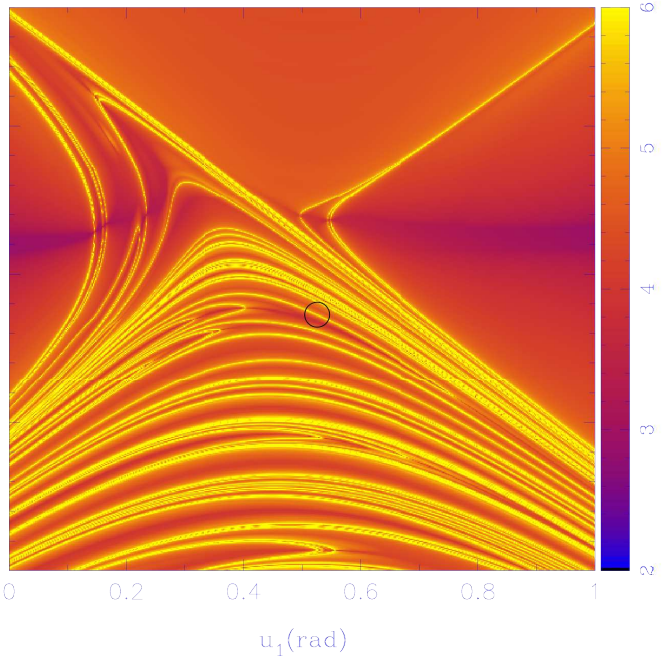

Molniya M1-8?

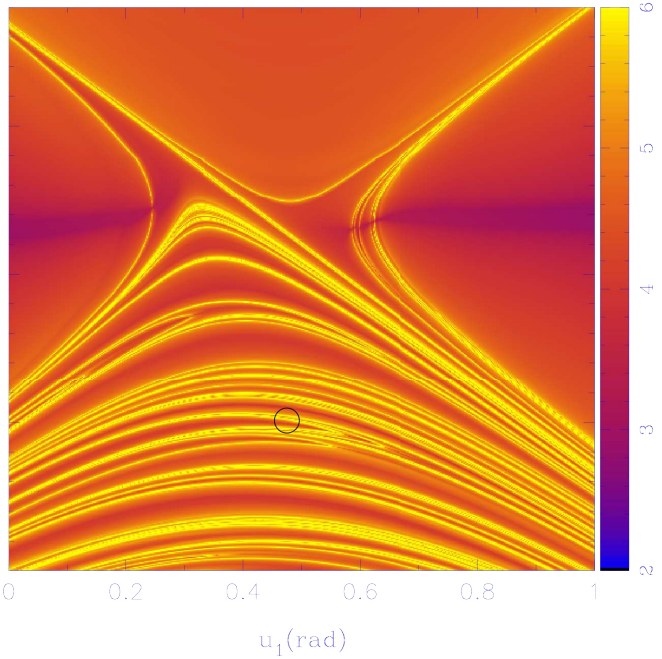

Fig. 7 Dynamical maps for Molniya M1-69 and M1-87. The locations of M1-69 and M1-87 are marked through the black circle. Both satellites reside within the hyperbolic tangle.

discussions with Ioannis Gkolias on the $J_{2}^{2}$ effect and useful references provided. J.D. acknowledges several discussions all along this research with Aaron Rosengren.

\section{Conflict of interest}

The authors declare that they have no conflict of interest.

\section{References}

1. Alessi, E.M., Buzzoni, A., Daquin, J., Carbognani, A., Tommei, G.: Dynamical properties of the molniya satellite constellation: Long-term evolution of orbital eccentricity. Acta Astronautica 179, 659-669 (2021)

2. Beletsky, V.V.: Essays on the motion of celestial bodies. Birkhäuser (2012)

3. Benest, D., Lega, E., Froeschlé, C.: Hamiltonian systems and fourier analysis: new prospects for gravitational dynamics. Cambridge Univ. Press (2005)

4. Breiter, S., Métris, G.: Symplectic mapping for satellites and space debris including nongravitational forces. Celestial Mechanics and Dynamical Astronomy 71(2), 79-94 (1998)

5. Breiter, S., Wytrzyszczak, I., Melendo, B.: Long-term predictability of orbits around the geosynchronous altitude. Advances in Space Research 35(7), 1313-1317 (2005)

6. Buzzoni, A., Guichard, J., Alessi, E.M., Altavilla, G., Figer, A., Carbognani, A., Tommei, G.: Spectrophotometric and dynamical properties of the soviet/russian constellation of molniya satellites. Journal of Space Safety Engineering 7(3), 255-261 (2020)

7. Capderou, M.: Handbook of satellite orbits: From kepler to GPS. Springer Science \& Business (2014)

8. Celletti, A., Galeş, C.: On the dynamics of space debris: 1: 1 and 2: 1 resonances. Journal of Nonlinear Science 24(6), 1231-1262 (2014)

9. Chirikov, B.V.: A universal instability of many-dimensional oscillator systems. Physics reports 52(5), 263-379 (1979)

10. Daquin, J., Deleflie, F., Pérez, J.: Comparison of mean and osculating stability in the vicinity of the (2: 1) tesseral resonant surface. Acta Astronautica 111, 170-177 (2015)

11. Daquin, J., Gkolias, I., Rosengren, A.J.: Drift and its mediation in terrestrial orbits. Frontiers in Applied Mathematics and Statistics 4, 35 (2018)

12. Delhaise, F., Henrard, J.: The problem of critical inclination combined with a resonance in mean motion in artificial satellite theory. Celestial Mechanics and Dynamical Astronomy 55(3), 261-280 (1993)

13. Delhaise, F., Morbidelli, A.: Luni-solar effects of geosynchronous orbits at the critical inclination. Celestial Mechanics and Dynamical Astronomy 57(1), 155-173 (1993)

14. Ely, T.A.: Dynamics and control of artificial satellite orbits with multiple tesseral resonances. Ph.D. thesis, Purdue University (1996)

15. Ely, T.A., Howell, K.C.: Dynamics of artificial satellite orbits with tesseral resonances including the effects of luni-solar perturbations. Dynamics and Stability of Systems 12(4), 243-269 (1997)

16. Ely, T.A., Howell, K.C.: East-west stationkeeping of satellite orbits with resonant tesseral harmonics. Acta Astronautica 46(1), 1-15 (2000) 


\section{A Dynamical model employed \& physical parameters}

As we mentioned in the introduction, the aim of this study is not to study with the greatest accuracy possible Molniya dynamics, with a comprehensive force model including uncertainty modeling and Monte Carlo like approaches. Quite on the contrary, we leverage the understanding of the dynamics of the semi-major axis from the essential "building blocks" with tractable contributions. In that respect, we would like to provide more context to the dynamical model we have employed. We have approached the problem as a drag-free model, with no solar radiation pressure, based on a compact geopotential model including relevant terms of the disturbing lunisolar potentials. Higher order zonal secular terms can be obtained in closed form over the eccentricity following the same formal procedure discussed in Section 2.1. In terms of the orbital elements, up to order $l=5$, they read:

$$
\left\{\begin{array}{l}
V_{J_{3}}=\frac{3 J_{3} \mu r_{\mathrm{E}}^{3} e \sin i\left(5 \sin ^{2} i-4\right)}{8 a^{4}\left(1-e^{2}\right)^{5 / 2}} \sin (\omega), \\
V_{J_{4}}=\left(\frac{15 J_{4} \mu r_{\mathrm{E}}^{4} \sin ^{2} i\left(7 \sin ^{2} i-6\right)}{64 a^{5}\left(1-e^{2}\right)^{7 / 2}}\right) \cos (2 \omega)-\frac{3 J_{4} \mu r_{\mathrm{E}}^{4}\left(3 e^{2}+2\right)\left(35 \sin ^{4} i-40 \sin ^{2} i+8\right)}{128 a^{5}\left(1-e^{2}\right)^{7 / 2}} \\
V_{J_{5}}=\left(\frac{15 J_{5} \mu r_{\mathrm{E}}^{5} e\left(3 e^{2}+4\right) \sin i\left(21 \sin ^{4} i-28 \sin ^{2} i+8\right)}{128 a^{6}\left(1-e^{2}\right)^{9 / 2}}\right) \sin (\omega)-\left(\frac{35 J_{5} \mu r_{\mathrm{E}}^{5} e^{3} \sin ^{3} i\left(9 \sin ^{2} i-8\right)}{256 a^{6}\left(1-e^{2}\right)^{9 / 2}}\right) \sin (3 \omega) .
\end{array}\right.
$$

It is worthwhile to note that the resonant argument of perigee also appears in the above secular contributions; hence the idea that Molniya orbits, besides tesseral and lunisolar resonances, gather also "zonal resonances". To include the second-order part term with factor $J_{2}^{2}$ in the secular Hamiltonian, with the form

$$
V_{J_{2}^{2}}=J_{2}^{2}(A(a, e, i) \cos 2 \omega+B(a, e, i)),
$$

we used the formula given in $[4,23]$. The relevance of our model $\mathcal{S}$ has been assessed by including those effects, and the lunisolar $h_{h}^{\mathrm{MS}} \cos h$ to $\mathcal{L}$. This model forms an "extended" Hamiltonian model $\tilde{\mathcal{S}}$. We computed the dynamical map for the Hamiltonian vector field $v_{\tilde{\mathcal{S}}}$ with $e=0.7, i_{0}=64^{\circ} 3$ and $(\omega, \Omega)=\left(270^{\circ}, 0\right)$ and we did not noticed significant macroscopic changes in the obtained dynamical template; henceforth the relevance of the Hamiltonian model $\mathcal{S}$. Let us mention that even if the macroscopic structures do not change drastically, hyperbolic orbits 


\begin{tabular}{lcc}
\hline \hline$k=\left(k_{1}, k_{2}\right)$ & $h_{k}(a, e, i)$ & $\sigma_{k}$ \\
\hline \hline$(1,0)$ & $\frac{\mu r_{\mathrm{E}}^{2} J_{21} 9 e \cos i \sin i}{4 a^{3}}$ & $M-\theta_{S}+\Omega$ \\
$(1,2)$ & $\frac{\mu r_{\mathrm{E}}^{2} J_{21} 3 e(\cos i+1) \sin i}{8 a^{3}}$ & $M-\theta_{S}+\Omega+2 \omega$ \\
$(2,0)$ & $\frac{-\mu r_{\mathrm{E}}^{2} J_{22} 27 e^{2}(\cos i+1)(\cos i-1)}{8 a^{3}}$ & $2\left(M-\theta_{S}+\Omega\right)$ \\
$(2,2)$ & $\frac{-\mu r_{\mathrm{E}}^{2} J_{22}\left(5 e^{2}-2\right)(\cos i+1)^{2}}{8 a^{3}}$ & $2\left(M-\theta_{S}+\Omega+\omega\right)$
\end{tabular}

\begin{tabular}{|c|c|c|}
\hline$(1,-1)$ & $\frac{\mu r_{\mathrm{E}}^{3} J_{31} 33 e^{2}\left(15 \cos i \sin ^{2} i-5 \sin ^{2} i-4 \cos i+4\right)}{128 a^{4}}$ & $M-\theta_{S}+\Omega-\omega$ \\
\hline$(1,1)$ & $\frac{-\mu r_{\mathrm{E}}^{3} J_{31} 3\left(2 e^{2}+1\right)\left(15 \cos i \sin ^{2} i-5 \sin ^{2} i-4 \cos i-4\right)}{16 a^{4}}$ & $M-\theta_{S}+\Omega+\omega$ \\
\hline$(1,3)$ & $\frac{\mu r_{\mathrm{E}}^{3} J_{31} 15 e^{2}(\cos i+1) \sin ^{2} i}{16 a^{4}}$ & $M-\theta_{S}+\Omega+\omega$ \\
\hline$(2,1)$ & $\frac{-\mu r_{\mathrm{E}}^{3} J_{32} 45 e(\cos i+1)(3 \cos i-1)}{8 a^{4}}$ & $2\left(M-\theta_{S}+\Omega\right)+\omega$ \\
\hline$(2,3)$ & $\frac{-\mu r_{\mathrm{E}}^{3} J_{32} 15 e(\cos i+1)^{2} \sin i}{8 a^{4}}$ & $2\left(M-\theta_{S}+\Omega\right)+3 \omega$ \\
\hline$(3,1)$ & $\frac{\mu r_{\mathrm{E}}^{3} J_{33} 2385 e^{2}(\cos i+1)^{2}(\cos i-1)}{64 a^{4}}$ & $3\left(M-\theta_{S}+\Omega\right)+\omega$ \\
\hline$(3,3)$ & $\frac{\mu r_{\mathrm{E}}^{3} J_{33} 15\left(6 e^{2}-1\right)(\cos i+1)^{3}}{8 a^{4}}$ & $3\left(M-\theta_{S}+\Omega\right)+3 \omega$ \\
\hline$(1,0)$ & $\frac{\mu r_{\mathrm{E}}^{4} J_{41} 75 e \cos i \sin i\left(7 \sin ^{2} i-4\right)}{16 a^{5}}$ & $M-\theta_{S}+\Omega$ \\
\hline$(1,2)$ & $\frac{-\mu r_{\mathrm{E}}^{4} J_{41} 5 e \sin i\left(14 \cos i \sin ^{2} i+7 \sin ^{2} i-6 \cos i-6\right)}{16 a^{5}}$ & $M-\theta_{S}+\Omega+2 \omega$ \\
\hline$(2,0)$ & $\frac{-\mu r_{\mathrm{E}}^{4} J_{42} 75 e^{2}\left(21 \cos ^{2} i \sin ^{2} i-7 \sin ^{2} i-4 \cos ^{2} i+4\right)}{8 a^{5}}$ & $2\left(M-\theta_{S}+\Omega\right)$ \\
\hline$(2,2)$ & $\frac{-\mu r_{\mathrm{E}}^{4} J_{42} 15\left(e^{2}+1\right)(\cos i+1)\left(7 \cos i \sin ^{2} i-\cos i-1\right)}{4 a^{5}}$ & $2\left(M-\theta_{S}+\Omega\right)+2 \omega$ \\
\hline$(2,4)$ & $\frac{-\mu r_{\mathrm{E}}^{4} J_{42} 105 e^{2}(\cos i+1)^{2} \sin ^{2} i}{32 a^{5}}$ & $2\left(M-\theta_{S}+\Omega\right)+4 \omega$ \\
\hline$(3,2)$ & $\frac{\mu r_{\mathrm{E}}^{4} J_{43} 945 e(\cos i+1)^{2}(2 \cos i-1) \sin i}{16 a^{5}}$ & $3\left(M-\theta_{S}+\Omega\right)+2 \omega$ \\
\hline$(3,4)$ & $\frac{\mu r_{\mathrm{E}}^{4} J_{43} 315 e(\cos i+1)^{3} \sin i}{32 a^{5}}$ & $3\left(M-\theta_{S}+\Omega\right)+4 \omega$ \\
\hline$(4,2)$ & $\frac{-\mu r_{\mathrm{E}}^{4} J_{44} 5565 e^{2}(\cos i-1)(\cos i+1)^{3}}{16 a^{5}}$ & $4\left(M-\theta_{S}+\Omega\right)+2 \omega$ \\
\hline$(4,4)$ & $\frac{-\mu r_{\mathrm{E}}^{4} J_{44} 105\left(11 e^{2}-1\right)(\cos i+1)^{4}}{16 a^{5}}$ & $4\left(M-\theta_{S}+\Omega\right)+4 \omega$ \\
\hline
\end{tabular}

Table 3 Formal coefficients and resonant angles of the $1: 1$ resonance for $l_{\max }=m_{\max }=4$ and up to $\mathcal{O}\left(e^{2}\right)$.

generated under model $\mathcal{S}$ and $\tilde{\mathcal{S}}$ will separate in time (sensitivity to the slight change of physics), and the hope to follow them beyond a few Lyapunov times is a useless effort. The Lyapunov time $\tau_{\mathcal{L}}$ computed as

$$
\left\{\begin{array}{l}
\chi=\lim _{s \rightarrow+\infty} \frac{1}{s} \log (\|w(s)\|), \\
\tau_{\mathcal{L}}=1 / \chi
\end{array}\right.
$$

is about 2 decades.

The physical parameters of this study read as follow. The Moon's orbital parameters, referred to the ecliptic plane, have been set to $a_{\mathrm{M}}=384,748 \mathrm{~km}, e_{\mathrm{M}}=0.0554, i_{\mathrm{M}}=5^{\circ} 15, \mu_{\mathrm{M}}=4902.8 \mathrm{~km}^{3} / \mathrm{s}^{2}$. The Sun's orbital parameters, referred to the Earth equator, have been set to $a_{\mathrm{S}}=1.496 \times 10^{8} \mathrm{~km}, e_{\mathrm{S}}=0.0167, i_{\mathrm{S}}=23^{\circ} 4392911$, $\mu_{\mathrm{S}}=1.32712 \times 10^{11} \mathrm{~km}^{3} / \mathrm{s}^{2}$. The length unit is the Earth radius $r_{\mathrm{E}}$ of $6378.1363 \mathrm{~km}, \mu=398,600.44 \mathrm{~km}^{3} / \mathrm{s}^{2}$.

\section{B Resonant potential for the geosynchronous tesseral resonance}

As mentioned, expressions presented in [8] related to the tesseral resonant contributions contain slight algebraic errors. We therefore present the algebraic expressions of the resonant tesseral associated to the geosynchronous region (1:1 resonance) up to order $l=4$. Orbits in this regime have smaller eccentricities and, as in [8], we truncate the development in eccentricity to $k_{\max }=2$. To derive the resonant contribution for the geosynchronous region, we follow identically the steps presented in subsection 2.2 apart that $u_{\mathrm{S}}$ reads now $u_{\mathrm{S}}=M-u_{\mathrm{F}}$, where $u_{\mathrm{F}}=\theta-\Omega$. Following our previous presentation, the formal coefficients and critical angles of $\mathcal{T}_{2}^{1: 1}, \mathcal{T}_{3}^{1: 1}, \mathcal{T}_{4}^{1: 1}$ are compactly summarised in Table 3 in terms of the Keplerian orbital elements. Let us recall that we denote by $\sigma_{k}$ the critical angle $\sigma_{k}=k_{1} u_{\mathrm{S}}+k_{2} \omega=k_{1}\left(M-\theta_{S}+\Omega\right)+k_{2} \omega$. 

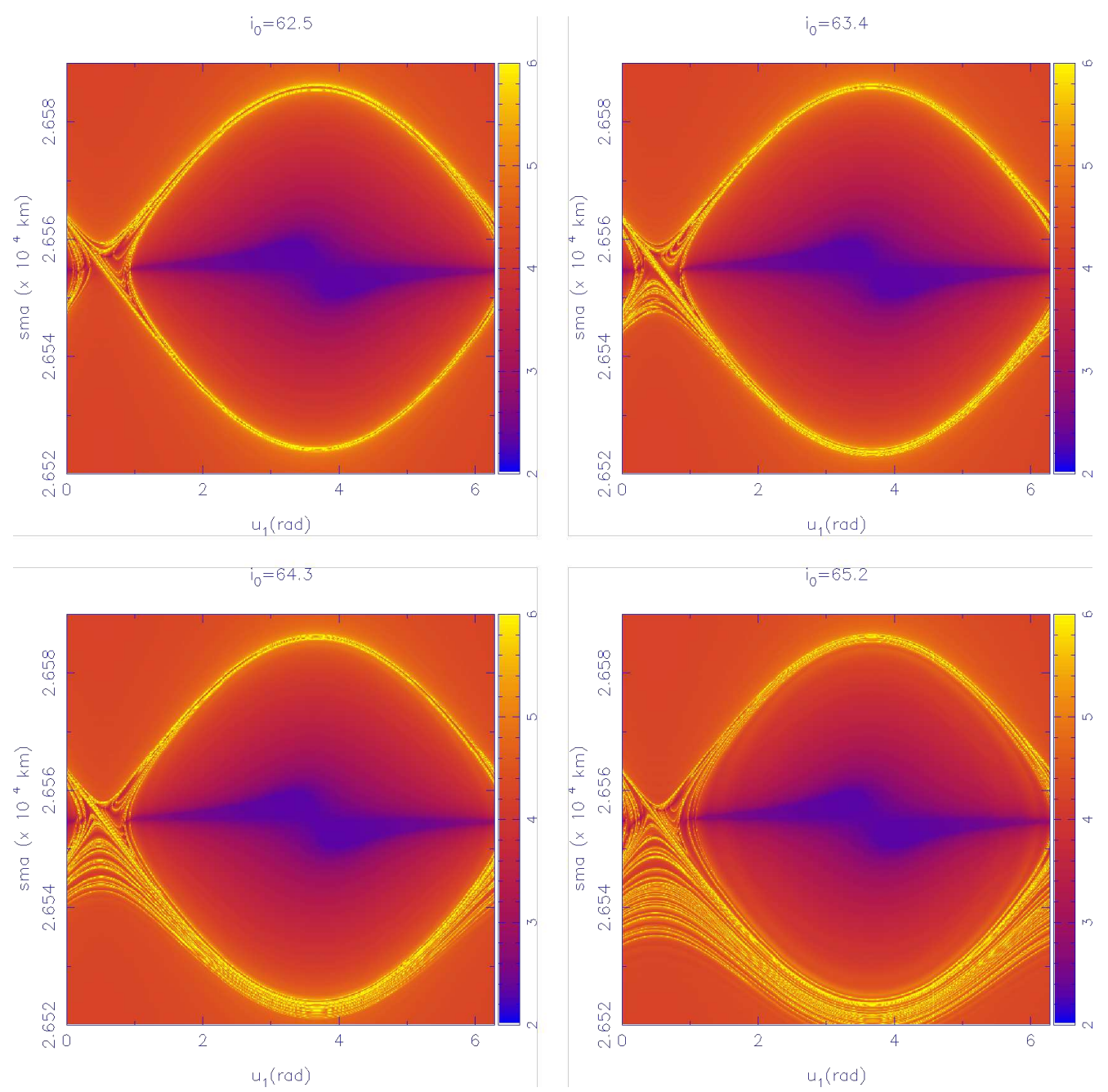

Fig. 8 Intersections of the forward in time FLIs with the plane $\left(a, u_{1}\right)$ for model $\mathcal{S}$ computed on a $500 \times 500$ grid of initial conditions for $i_{0} \in\{62.5,63.4,64.3,65.2\} \mathrm{deg}$.

\section{5}

386

\section{Dynamical maps}

We computed dynamical maps for a fixed value of $e=0.7$ and $i_{0}$ "piercing" the critical inclination. They are presented in Fig. 8 for model $\mathcal{S}$. Given that model $\mathcal{J}$ is slightly perturbed, we just show the maps for $i_{0}=62^{\circ} 5$ and $i_{0}=65^{\circ} 2$ in Fig. 9. The maps have been computed on a $500 \times 500$ grid of initial conditions, forward in time, and over a time interval of 20 years. We have considered 4 values of the initial inclinations, namely $i_{0} \in\left\{62^{\circ} 5,63^{\circ} 4,64^{\circ} 3,65^{\circ} 2\right\}$. The initial phasing is set as $(\omega, \Omega)=\left(270^{\circ}, 0^{\circ}\right)$. If a given initial condition in the map fall within the highest region of the FLIs (yellow tone), then the orbit is hyperbolic and exhibit sensitive dependence upon the initial condition (i.e., any orbit starting with an initial condition slightly different will have a long-term different future; the orbits will separate with time). We note that the $i_{0}$-dependence of the $\mathcal{J}$ model is quasi-absent. The model $\mathcal{J}$ is very close to the integrable picture, in the sense that the splitting of the separatrix is weak. The latter is much more manifest for model $\mathcal{S}$, where we recall, the eccentricity and inclinations variables are no longer frozen. For increasing values of $i_{0}$, we underline the growing asymmetry of the foldings near the saddle-like structure for the model $\mathcal{S}$. This particular structure transfers directly at the single orbit level: an orbit trapped within the hyperbolic layer is more likely to display U-turns intermittency phenomena towards the lower semi-major axis. This observation, based on the thin structures of the lobes detected with a variational dynamical indicator on our model, is also in agreement with the actual two-line elements datasets for objects M1-69 and M1-87. 


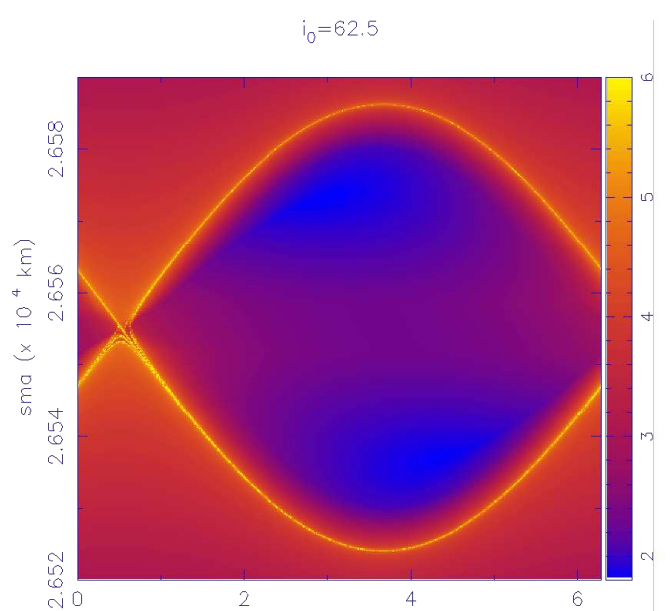

$u_{1}(\mathrm{rad})$

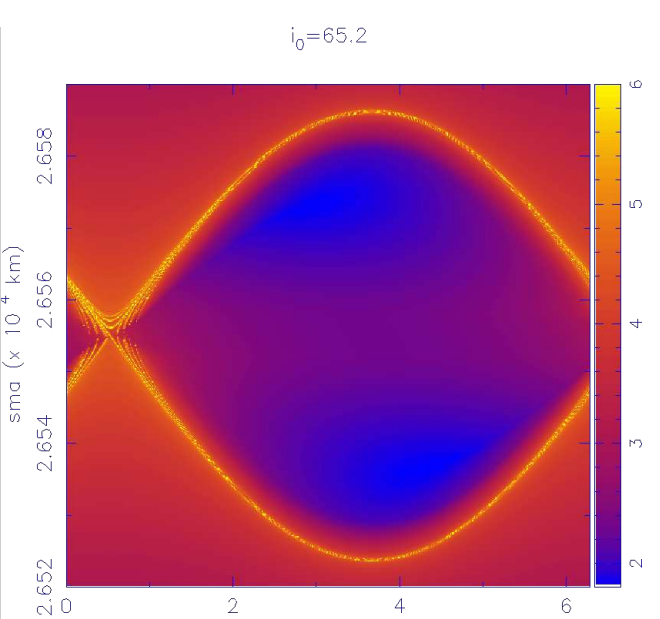

$u_{1}(\mathrm{rod})$

Fig. 9 Intersections of the forward in time FLIs with the plane $\left(a, u_{1}\right)$ for model $\mathcal{J}$ computed on a $500 \times 500$ grid of initial conditions for $i_{0} \in\{62.5,65.2\} \mathrm{deg}$. 
Figures

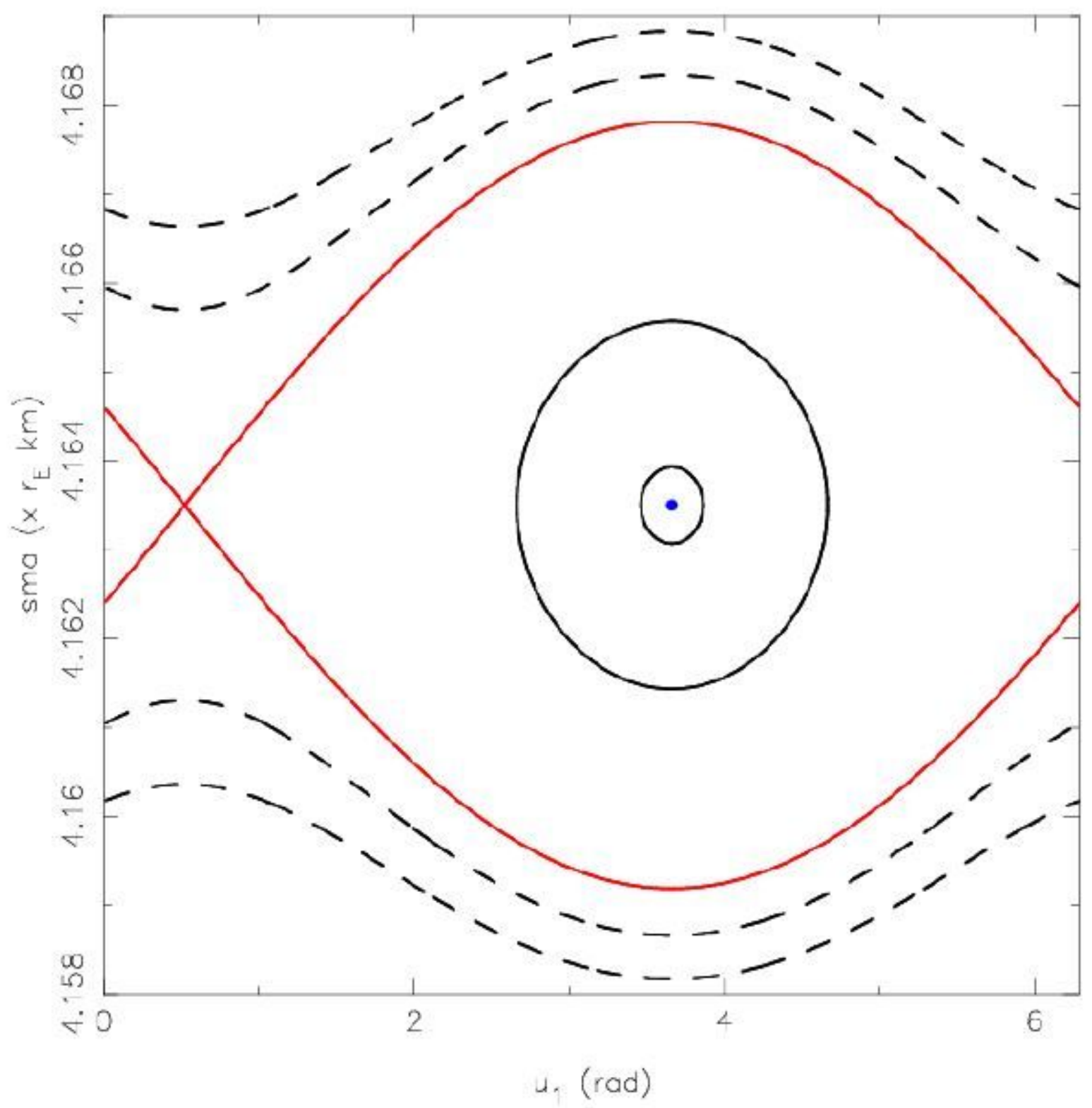

Figure 1

Phase space of the resonant integrable approximation. The width of the separatrix (red curve) allows excursion of the semi-major axis up to $2 \Delta \mathrm{a}=54 \mathrm{~km}$ within the libration domain. The oscillations near the elliptic xed-point (blue point) have a period of about 1:76 years. 

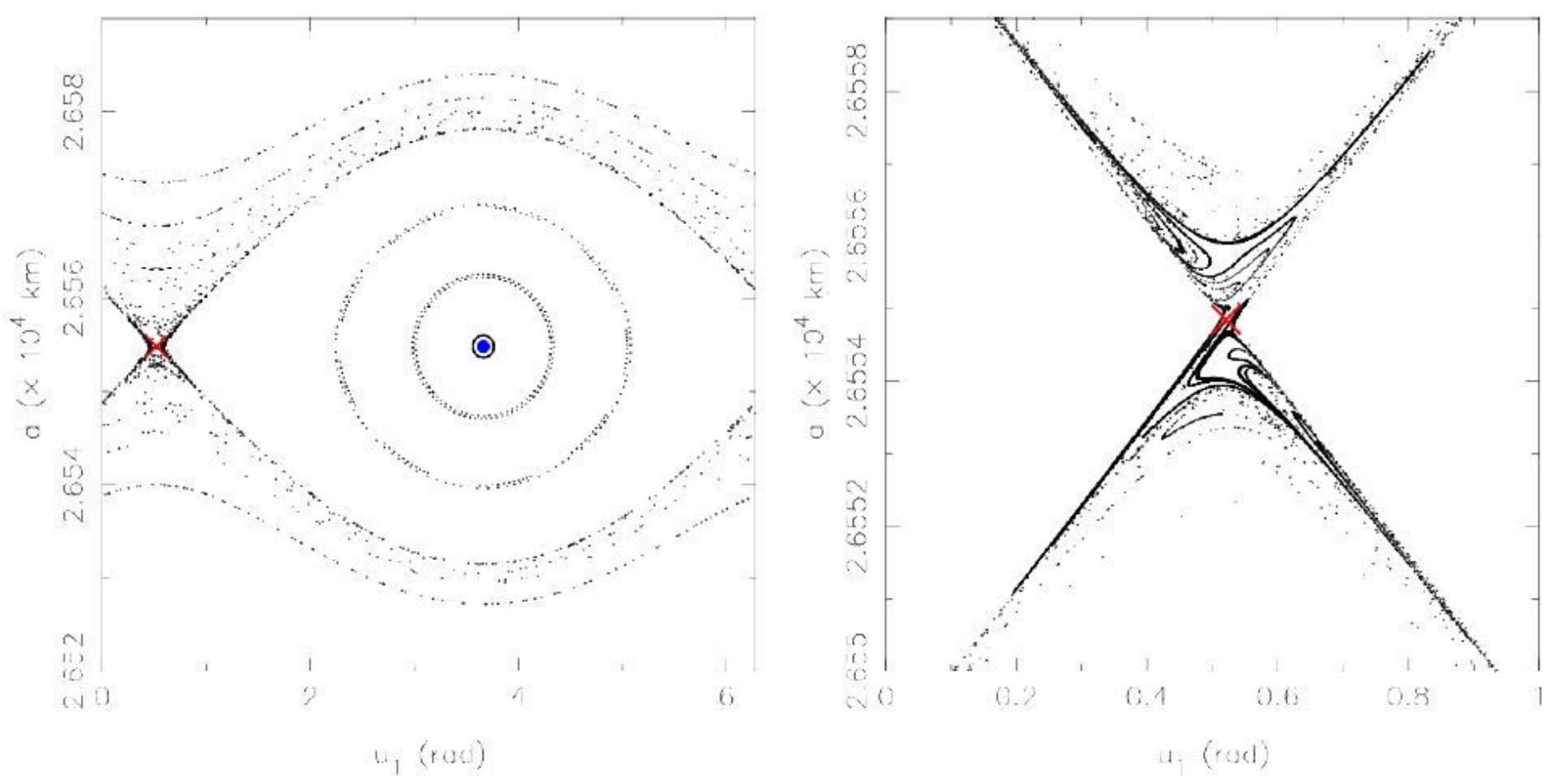

Figure 2

(Left) Poincare section associated to (35) computed for $\mathrm{g}(0)=0$. The unstable xed point is labeled with the red cross, the blue circle surrounds the stable periodic orbit. The phase space is similar to the integrable approximation but contains a thin chaotic layer (scattered erratic points) surrounding the unperturbed separatrix. Each considered initial condition has been iterated 100 times under P. (Right) Details of nite pieces of the stable manifold Ws(xu). 

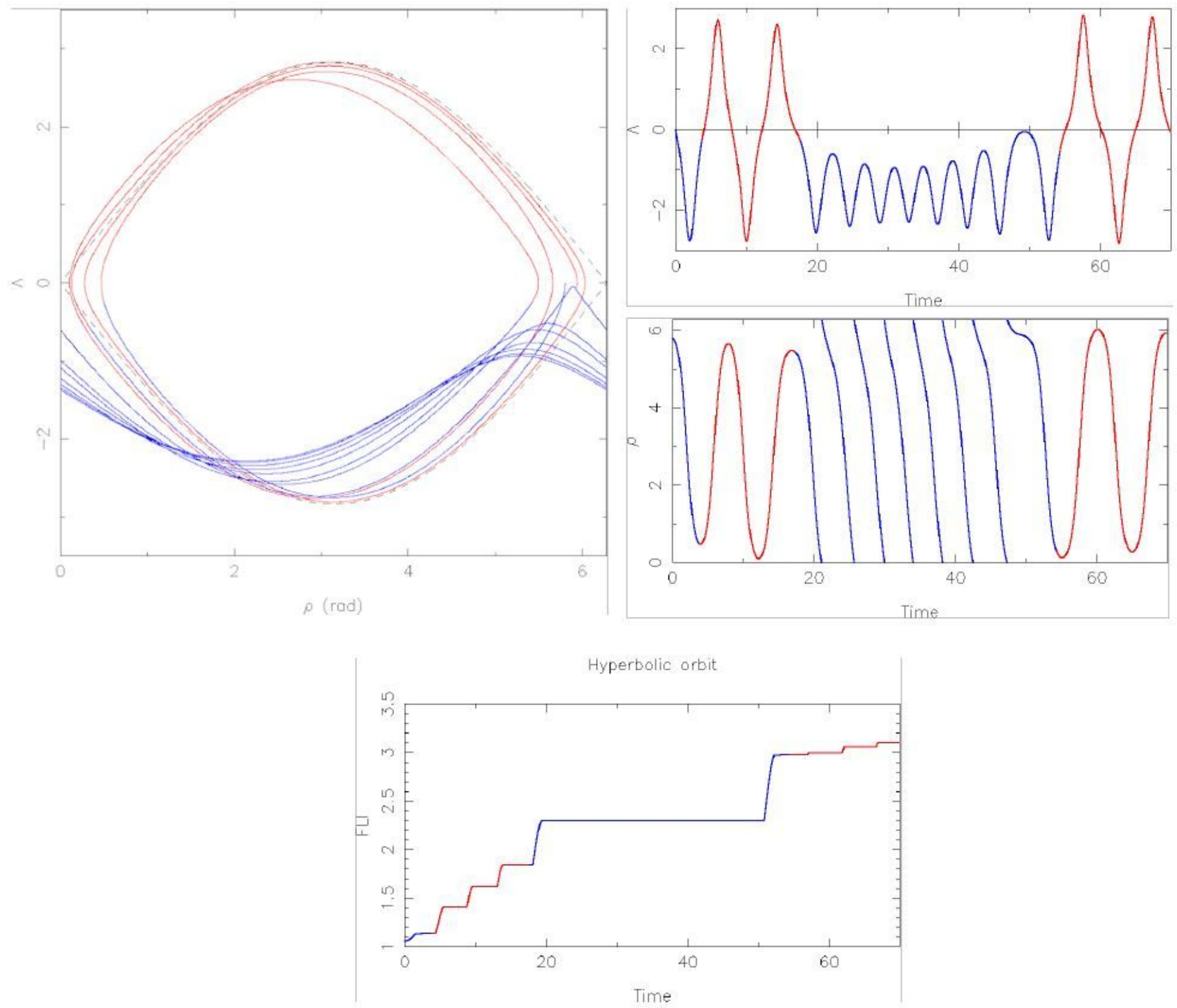

Figure 3

Composite plot illustrating the mechanisms of the intermittency phenomena. The dashed black line represents the separatrix of the integrable model $\mathrm{T}$. Realisations of the stable and unstable manifolds, for $\nabla 6 \neq 0 ; \otimes<<1$, are not shown for the sake of readability. One hyperbolic orbit trapped in the hyperbolic tangle is highlighted in the phase space, with a color code depending on the regime of the resonant angle. When the resonant angle circulates (blue color), $(p) \neq 0$, the action takes negative $\Delta$ 's. When the angle librates (red color), ( $p) \sim 0$, the action variable performs the full homoclinic loop and exhibit larger variations. Alternation between circulation and librations with passage close to the saddle contribute to breaks the plateau structure of the FLI and produces jumps in the FLI. 

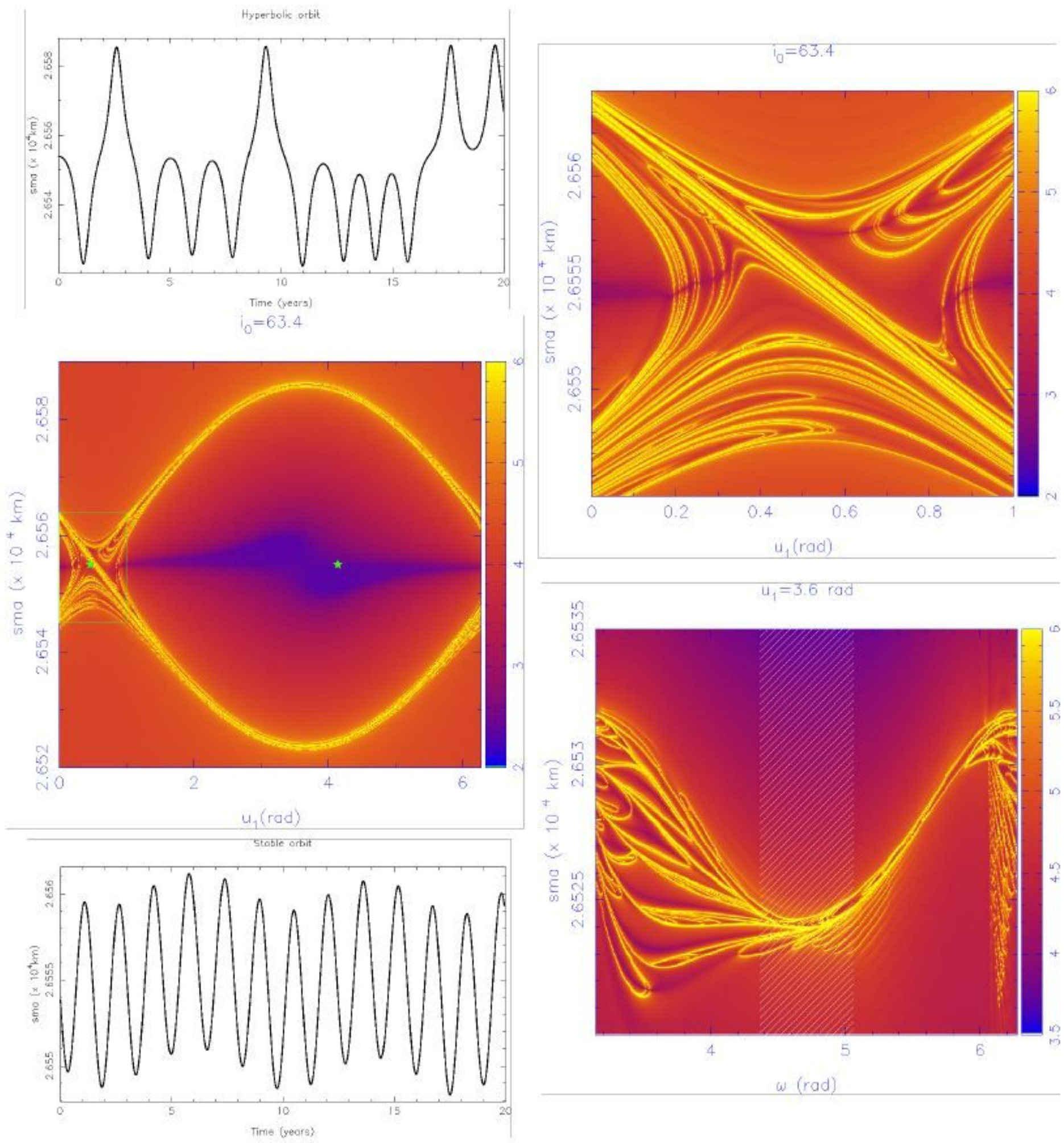

\section{Figure 4}

Composite plot highlighting the main features of Molniya semi-major axis dynamics. The global FLI map and a magnied portion near the saddle-like structure detail the hyperbolic structure. Initial conditions within the hyperbolic layer display intermittency phenomena, whilst stable orbits display regular oscillations. This is exemplied for two orbits whose initial conditions are labeled with the green stars. The width of the layer, for a xed u1 but varying $\omega$, might exhibit a complex geometry. For Molniya's 
prototypical range of values of $\omega$, materialised by the white shaded-line region around $\omega=270^{\circ}$, the width is limited to a few kilometer in the semi-major axis only. See text for details.
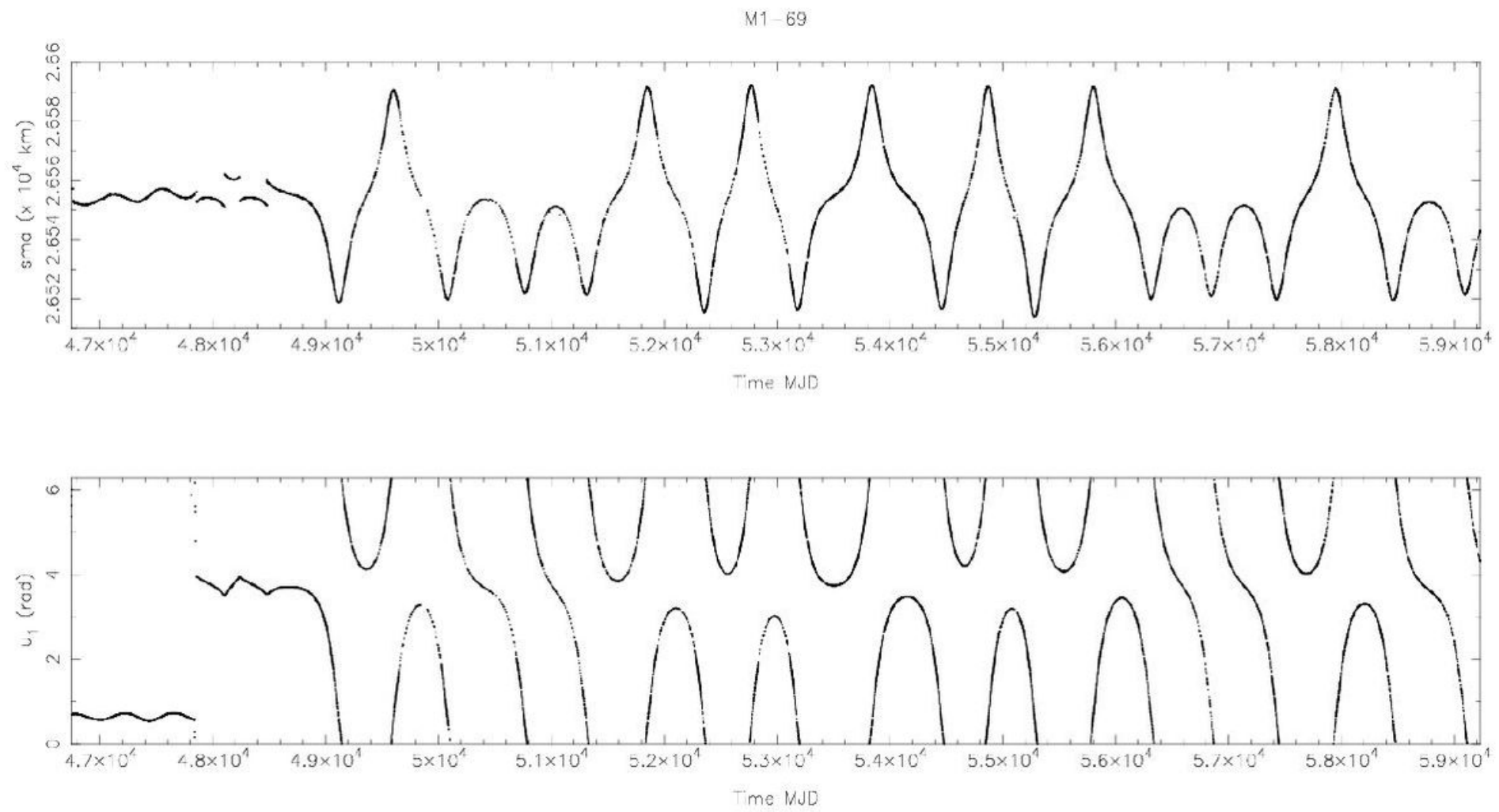

Figure 5

Time history of the semi-major axis and resonant angle $u 1$ extracted from the TLE data for the satellite M1-69.
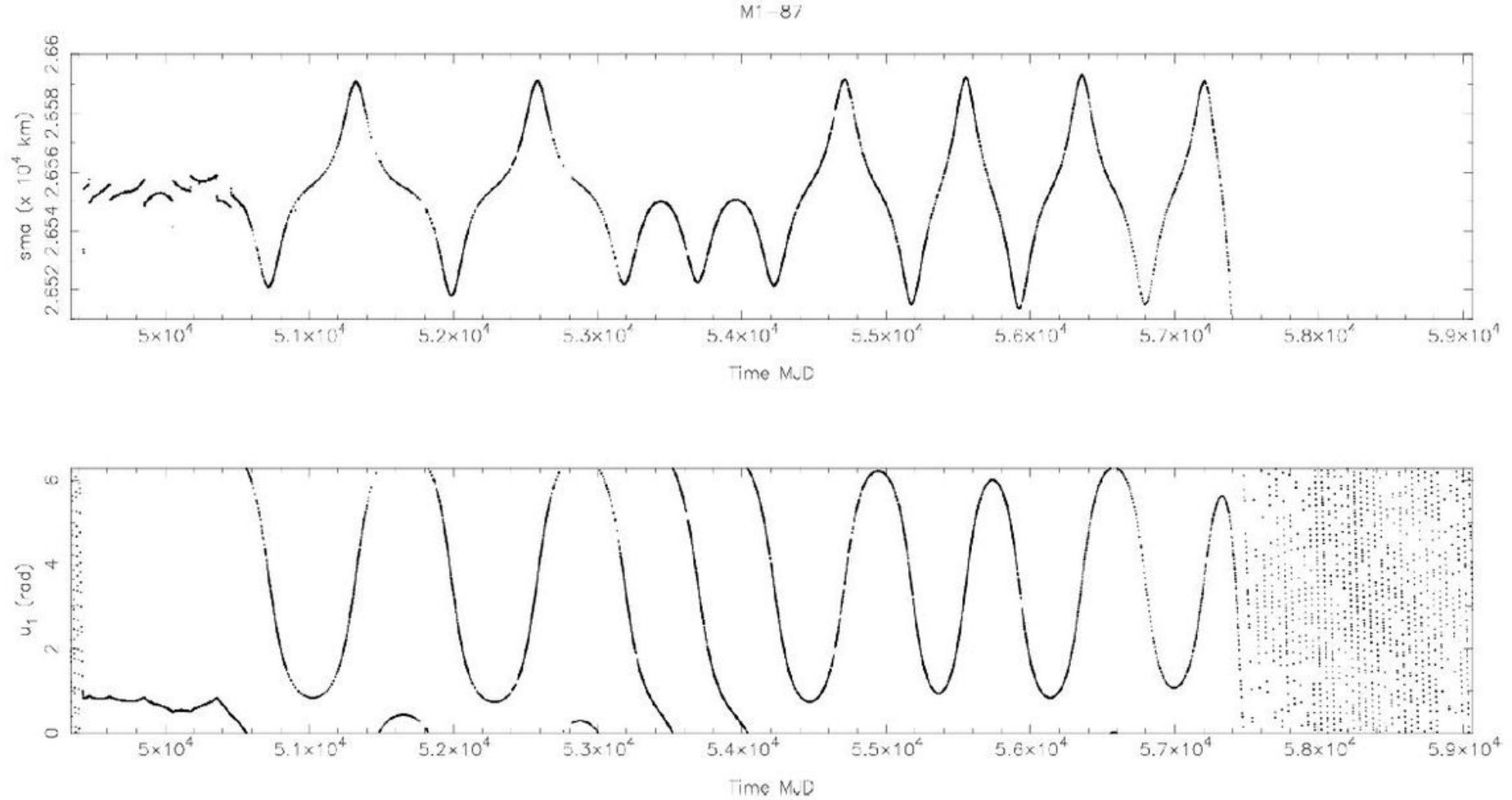


\section{Figure 6}

Time history of the semi-major axis and resonant angle u1 extracted from the TLE data for the satellite M1-87

Wolniya V1-69

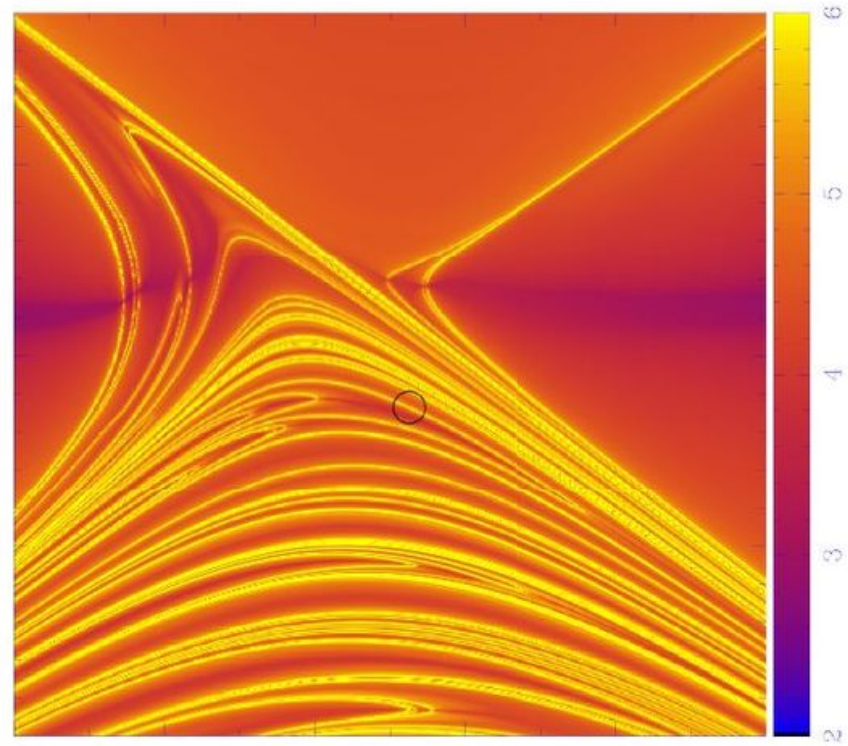

$u_{1}(\mathrm{raci})$
Molniya M1-87

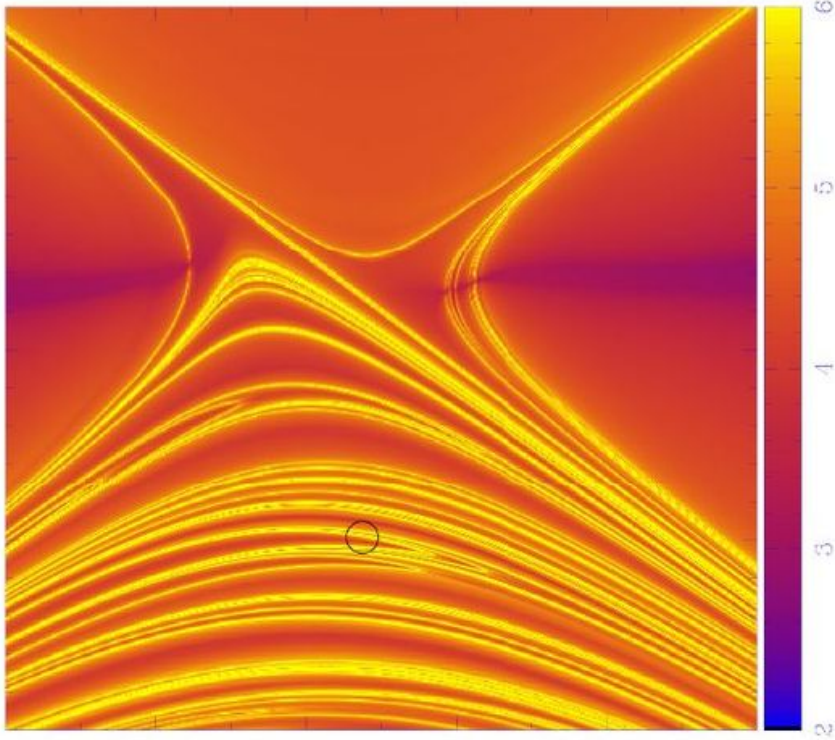

U. $(\mathrm{rad})$

\section{Figure 7}

Dynamical maps for Molniya M1-69 and M1-87. The locations of M1-69 and M1-87 are marked through the black circle. Both satellites reside within the hyperbolic tangle. 

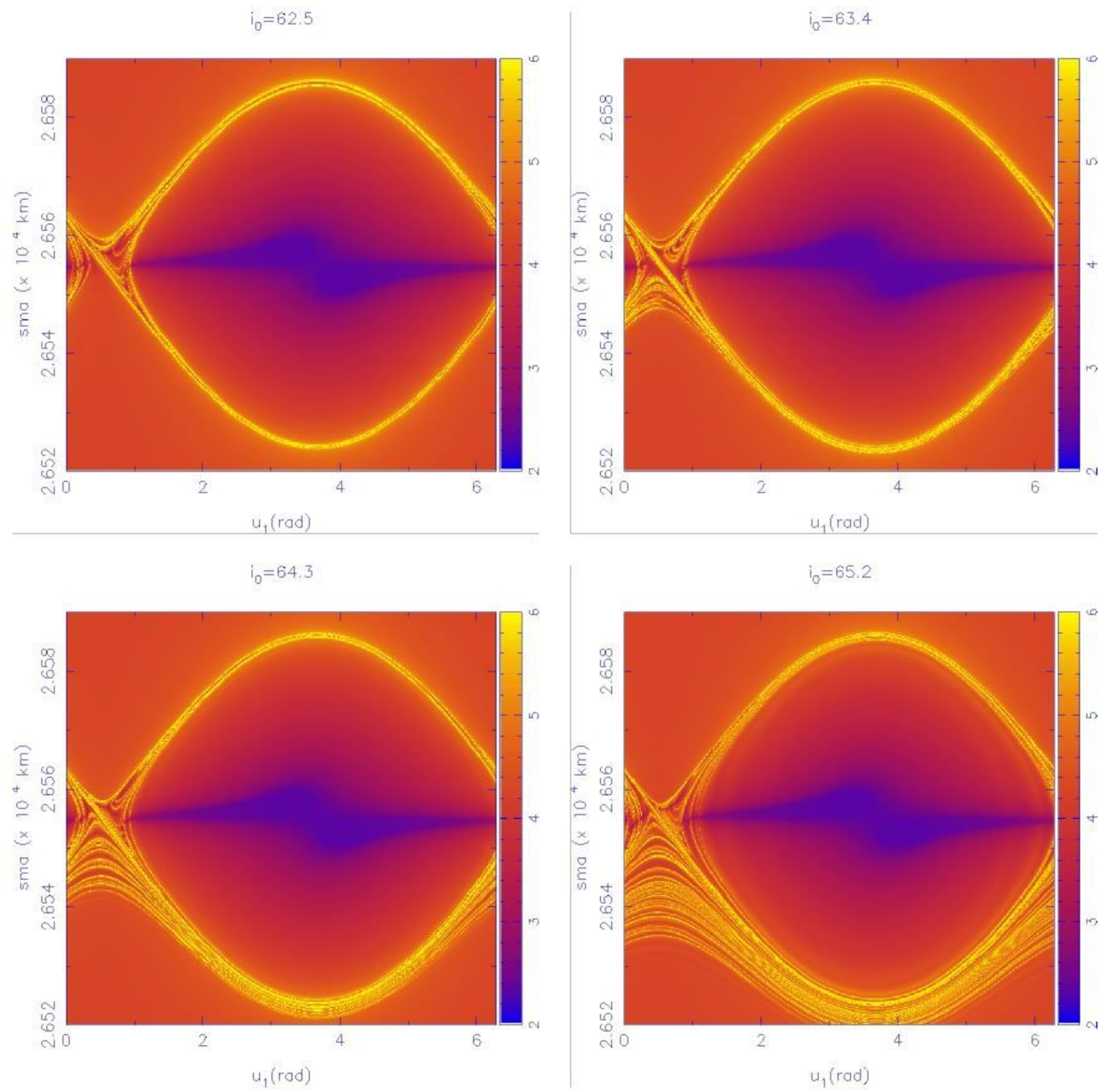

\section{Figure 8}

Intersections of the forward in time FLIs with the plane (a; u1) for model S computed on a $500 \times 500$ grid

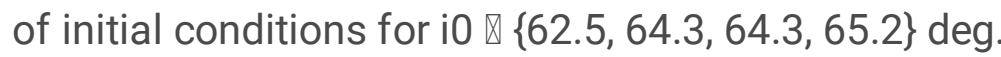



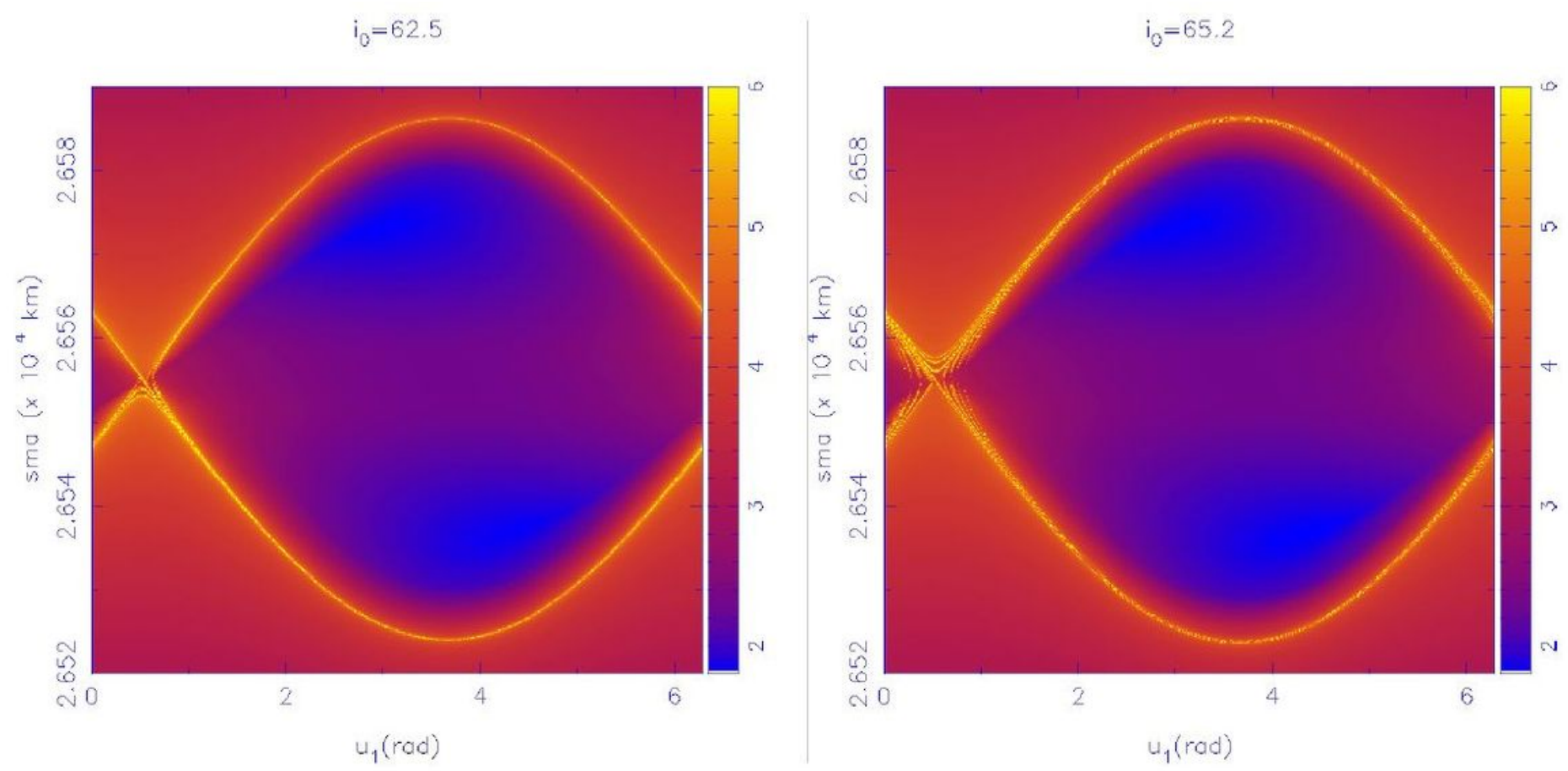

Figure 9

Intersections of the forward in time FLIs with the plane (a; u1) for model $\mathrm{J}$ computed on a $500 \times 500$ grid

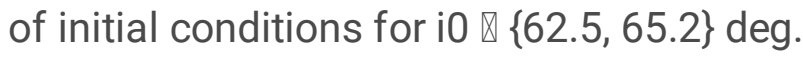

